\title{
ON THE DEFINING IDEAL OF A SET OF POINTS IN MULTI-PROJECTIVE SPACE
}

\author{
ADAM VAN TUYL
}

\begin{abstract}
We investigate the defining ideal $I_{\mathbb{X}}$ of a set of points $\mathbb{X}$ in $\mathbb{P}^{n_{1}} \times \cdots \times \mathbb{P}^{n_{k}}$ with a special emphasis on the case that $\mathbb{X}$ is in generic position, that is, $\mathbb{X}$ has the maximal Hilbert function. When $\mathbb{X}$ is in generic position, we determine the degrees of the generators of the associated ideal $I_{\mathbb{X}}$. Letting $\nu\left(I_{\mathbb{X}}\right)$ denote the minimal number of generators of $I_{\mathbb{X}}$, we use this description of the degrees to construct a function $v\left(s ; n_{1}, \ldots, n_{k}\right)$ with the property that $\nu\left(I_{\mathbb{X}}\right) \geq v\left(s ; n_{1}, \ldots, n_{k}\right)$ always holds for $s$ points in generic position in $\mathbb{P}^{n_{1}} \times \cdots \times \mathbb{P}^{n_{k}}$. When $k=1, v\left(s ; n_{1}\right)$ equals the expected value for $\nu\left(I_{\mathbb{X}}\right)$ as predicted by the Ideal Generation Conjecture. If $k \geq 2$, we show that there are cases with $\nu\left(I_{\mathbb{X}}\right)>v\left(s ; n_{1}, \ldots, n_{k}\right)$. However, computational evidence suggests that in many cases $\nu\left(I_{\mathbb{X}}\right)=v\left(s ; n_{1}, \ldots, n_{k}\right)$.
\end{abstract}

\section{INTRODUCTION}

In this paper we investigate the generators of the ideal $I_{\mathbb{X}}$ defining a set of points $\mathbb{X}$ in generic position in $\mathbb{P}^{n_{1}} \times \cdots \times \mathbb{P}^{n_{k}}$.

One of the fundamental open problems about finite sets of points $\mathbb{X} \subseteq \mathbb{P}^{n}$ in generic position, i.e., those sets of points having the maximal Hilbert function, is to count the minimal number of generators of $I_{\mathbb{X}}$ in terms of the data $n$ and $|\mathbb{X}|=s$. This question is the content of the Ideal Generation Conjecture (IGC) (see [7). Recently, many authors (cf. 2, 8, 9, 10, 11, 12, 16, 17) have been interested in generalizing results about points in $\mathbb{P}^{n}$ to $\mathbb{P}^{n_{1}} \times \cdots \times \mathbb{P}^{n_{k}}$. We continue this program by studying the generators of $I_{\mathbb{X}}$ when $\mathbb{X} \subseteq \mathbb{P}^{n_{1}} \times \cdots \times \mathbb{P}^{n_{k}}$ with the hope that this might lead to a generalized IGC. Our investigation was also partially motivated by the desire to understand which properties about the ideal of points in $\mathbb{P}^{n}$, specifically those shown in [4, 6, 7, carry over to $\mathbb{P}^{n_{1}} \times \cdots \times \mathbb{P}^{n_{k}}$.

Given the defining ideal $I_{\mathbb{X}}$ of a set of points $\mathbb{X} \subseteq \mathbb{P}^{n_{1}} \times \cdots \times \mathbb{P}^{n_{k}}$, two natural questions about the generators of $I_{\mathbb{X}}$ arise: (1) what are the degrees of the generators? and (2) what is $\nu\left(I_{\mathbb{X}}\right):=$ minimal number of generators of $I_{\mathbb{X}}$ ? These questions can be viewed as the first step in describing the multi-graded minimal free resolution of $I_{\mathbb{X}}$ since (1) and (2) are questions about the 0th multi-graded Betti numbers.

In Section 2 we show that the Hilbert function of a set of points can be used to bound the degrees of the generators, thus giving a partial answer to (1). As posed, however, these questions are difficult to attack, even when $k=1$, without further conditions on the points.

For finite sets of points $\mathbb{X} \subseteq \mathbb{P}^{n}$, these questions have been primarily studied under the extra hypothesis that the set of points is in generic position, i.e., $H_{\mathbb{X}}(i)=\min \left\{\operatorname{dim}_{\mathbf{k}} R_{i},|\mathbb{X}|\right\}$ for all $i \in \mathbb{N}$. Thus, one is led to ask about the generators of $I_{\mathbb{X}}$ when $\mathbb{X}$ is a set of points in generic position in $\mathbb{P}^{n_{1}} \times \cdots \times \mathbb{P}^{n_{k}}$. However, it is first necessary to establish the basic properties (like existence) of points in generic position in multi-projective spaces since these facts are not part of the literature. Analogous to the case of points in $\mathbb{P}^{n}$, we say that a set $\mathbb{X}$ of $s$ points in $\mathbb{P}^{n_{1}} \times \cdots \times \mathbb{P}^{n_{k}}$ is in generic position if $H_{\mathbb{X}}(\underline{i})=\min \left\{\operatorname{dim}_{\mathbf{k}} R_{\underline{i}}, s\right\}$ for all $\underline{i} \in \mathbb{N}^{k}$. In Section 3 we show that these points exist, and moreover, if we consider each set of $s$ points as a point in $\left(\mathbb{P}^{n_{1}} \times \cdots \times \mathbb{P}^{n_{k}}\right)^{s}$, the points in generic position form a non-empty open subset of $\left(\mathbb{P}^{n_{1}} \times \cdots \times \mathbb{P}^{n_{k}}\right)^{s}$ with respect to the Zariski topology.

2000 Mathematics Subject Classification. 13D02,13D40, 14A05.

Key words and phrases. points, multi-projective space, generators.

Supported in part by the Natural Sciences and Engineering Research Council of Canada. 
We also show in Section 3 that if $F$ is a generator of $I_{\mathbb{X}}$, then $\operatorname{deg} F=\underline{i}$ or $\underline{i}+e_{j}$ where $\underline{i} \in \mathcal{D}:=$ $\left.\min \left\{\underline{i} \in \mathbb{N}^{k} \mid \begin{array}{c}i_{1}+n_{1} \\ i_{1}\end{array}\right) \cdots\left(\begin{array}{c}i_{k}+n_{k} \\ i_{k}\end{array}\right)>s\right\}$ and $e_{j}$ is one of the $k$ basis vectors of $\mathbb{N}^{k}$. This result gives an answer to (1) and generalizes the fact that $I_{\mathbb{X}}=\left\langle\left(I_{\mathbb{X}}\right)_{d} \oplus\left(I_{\mathbb{X}}\right)_{d+1}\right\rangle$ with $d=\min \left\{i \mid\left(\begin{array}{c}i+n \\ i\end{array}\right)>s\right\}$ in the graded case. An interesting difference between points in generic position in $\mathbb{P}^{n}$ versus $\mathbb{P}^{n_{1}} \times \cdots \times \mathbb{P}^{n_{k}}$ is that $R / I_{\mathbb{X}}$ is always Cohen-Macaulay if $k=1$, but is never Cohen-Macaulay if $k \geq 2$ (see Theorem 3.4)

In Section 4 we use this description of the degrees to show that $\nu\left(I_{\mathbb{X}}\right)$ can be determined by counting the generators of degree $\underline{i}$ and $\underline{i}+e_{j}$ for all $\underline{i} \in \mathcal{D}$ and $j=1, \ldots, k$. By degree considerations, $I_{\mathbb{X}}$ has $\operatorname{dim}_{\mathbf{k}}\left(I_{\mathbb{X}}\right)_{\underline{i}}$ generators of degree $\underline{i}$ for each $\underline{i} \in \mathcal{D}$. To count the generators of degree $\underline{i}+e_{j}$, we need to calculate the dimension of the image of the map $\Phi_{\underline{i}, j}: R_{e_{j}} \otimes_{\mathbf{k}}\left(I_{\mathbb{X}}\right)_{\underline{i}} \stackrel{a \times b}{\longrightarrow}\left(I_{\mathbb{X}}\right)_{\underline{i}+e_{j}}$ for each $\underline{i} \in \mathcal{D}$ and $1 \leq j \leq k$. Moreover, if there exists $\underline{i}_{1}, \underline{i}_{2} \in \mathcal{D}$ and $1 \leq j_{1}, j_{2} \leq k$ such that $l=\underline{i}_{1}+e_{j_{1}}=\underline{i}_{2}+e_{j_{2}}$, then $\operatorname{Im} \Phi_{\underline{i}_{1}, e_{j_{1}}}$ and $\operatorname{Im} \Phi_{\underline{i}_{1}, e_{j_{2}}}$ are both subspaces of $\left(I_{\mathbb{X}}\right)_{l}$, so we also need to know $\operatorname{dim}_{\mathbf{k}}\left(\operatorname{Im}_{\underline{i}_{1}, e_{j_{1}}} \cap \operatorname{Im} \Phi_{\underline{i}_{1}, e_{j_{2}}}\right)$. In general, it is difficult to compute the sizes of these vector spaces, even if $k=1$, except in some special cases. When $k=1$, to compute $\nu\left(I_{\mathbb{X}}\right)$ only the dimension of the image of $\Phi: R_{1} \otimes_{\mathbf{k}}\left(I_{\mathbb{X}}\right)_{d} \rightarrow\left(I_{\mathbb{X}}\right)_{d+1}$ needs to be calculated. The IGC states that $\operatorname{Im} \Phi$ should be as large as possible for a sufficiently general set of points (a subset of those points in generic position).

By considering the largest possible value for each $\operatorname{dim}_{\mathbf{k}} \operatorname{Im} \Phi_{i, j}$, in Section 5 we construct a function $v\left(s ; n_{1}, \ldots, n_{k}\right)$ with the property that $\nu\left(I_{\mathbb{X}}\right) \geq v\left(s ; n_{1}, \ldots, n_{k}\right)$ always holds for a set of $s$ points in generic position in $\mathbb{P}^{n_{1}} \times \cdots \times \mathbb{P}^{n_{k}}$. When $k=1, v(s ; n)$ equals the expected value for $\nu\left(I_{\mathbb{X}}\right)$ as predicted by the IGC. Furthermore, using [9], 10, we show that $\nu\left(I_{\mathbb{X}}\right)=v(s ; 1,1)$ for a sufficiently general set of $s$ points in $\mathbb{P}^{1} \times \mathbb{P}^{1}$.

Buoyed by these results, we had hoped that for any set of $s$ points in generic position that were sufficiently general, we should expect $\nu\left(I_{\mathbb{X}}\right)=v\left(s ; n_{1}, \ldots, n_{k}\right)$. However, we show that if $\mathbb{X}$ is any three points in generic position in $\mathbb{P}^{1} \times \cdots \times \mathbb{P}^{1}(k \geq 3$ times $)$, then $\nu\left(I_{\mathbb{X}}\right)>v(3 ; 1, \ldots, 1)$. As well, computational evidence suggests that $\nu\left(I_{\mathbb{X}}\right)>v(s ; 1, n, n)$ if $s=1+n+n$. These cases appear to be exceptional because in all other computed examples the equality $\nu\left(I_{\mathbb{X}}\right)=v\left(s ; n_{1}, \ldots, n_{k}\right)$ holds. Moreover, we know of no example of $\nu\left(I_{\mathbb{X}}\right)>v\left(s ; n_{1}, n_{2}\right)$ when $k=2$. This leads us to believe that $\nu\left(I_{\mathbb{X}}\right)=v\left(s ; n_{1}, \ldots, n_{k}\right)$ in a large number of cases, thus giving us a partial generalization of the the IGC.

\section{Preliminaries}

In this paper $\mathbf{k}$ denotes a field with $\operatorname{char}(\mathbf{k})=0$ and $\mathbf{k}=\mathbf{k}$. This section provides the relevant facts and definitions about multi-graded rings, Hilbert functions, and sets of points in multi-projective spaces. See also [15, 16, 17].

Let $\mathbb{N}:=\{0,1,2, \ldots\}$. For any integer $k \geq 1$, we write $[k]:=\{1, \ldots, k\}$. We denote $\left(i_{1}, \ldots, i_{k}\right) \in \mathbb{N}^{k}$ by $\underline{i}$. We set $|\underline{i}|:=\sum_{h} i_{h}$. If $\underline{i}, \underline{j} \in \mathbb{N}^{k}$, then $\underline{i}+\underline{j}:=\left(i_{1}+j_{1}, \ldots, i_{k}+j_{k}\right)$. We write $\underline{i} \geq \underline{j}$ if $i_{h} \geq j_{h}$ for every $h=1, \ldots, k$. Observe that $\geq$ is a partial order on $\mathbb{N}^{k}$. For any subset $\mathcal{A} \subseteq \mathbb{N}^{k}$, we will use $\min \mathcal{A}$ to denote the set of minimal elements of $\mathcal{A}$ with respect to this partial order. The set $\mathbb{N}^{k}$ is a semi-group generated by $\left\{e_{1}, \ldots, e_{k}\right\}$ where $e_{i}:=(0, \ldots, 1 \ldots, 0)$ is the $i$ th standard basis vector of $\mathbb{N}^{k}$. For any $c \in \mathbb{N}, c e_{i}:=(0, \ldots, c, \ldots, 0)$.

Set $R=\mathbf{k}\left[x_{1,0}, \ldots, x_{1, n_{1}}, x_{2,0}, \ldots, x_{2, n_{2}}, \ldots, x_{k, 0}, \ldots, x_{k, n_{k}}\right]$, and induce an $\mathbb{N}^{k}$-grading on $R$ by setting $\operatorname{deg} x_{i, j}=e_{i}$. An element $x \in R$ is said to be $\mathbb{N}^{k}$-homogeneous (or simply homogeneous if the grading is clear) if $x \in R_{\underline{i}}$ for some $\underline{i} \in \mathbb{N}^{k}$. If $x$ is homogeneous, then $\operatorname{deg} x:=\underline{i}$.

An ideal $I=\left(F_{1}, \ldots, F_{r}\right) \subseteq R$ is an $\mathbb{N}^{k}$-homogeneous (or, simply homogeneous) ideal if each $F_{j}$ is $\mathbb{N}^{k}$-homogeneous. If $I \subseteq R$ is a homogeneous ideal, $S=R / I$ inherits an $\mathbb{N}^{k}$-graded ring structure if we define $S_{\underline{i}}=(R / I)_{\underline{i}}:=R_{\underline{i}} / I_{\underline{i}}$. The function $H_{S}(\underline{i}):=\operatorname{dim}_{\mathbf{k}}(R / I)_{\underline{i}}$ is the Hilbert function of $S$.

If $I$ is an $\mathbb{N}^{k}$-homogeneous ideal of $R$, then for any $\underline{i} \in \mathbb{N}^{k}$, and for any $j \in[k]$, we set

$$
R_{e_{j}} I_{\underline{i}}:=\left\{f \mid f=f_{0} x_{j, 0}+f_{1} x_{j, 1}+\cdots+f_{n_{j}} x_{j, n_{j}}, f_{l} \in I_{\underline{i}}\right\} .
$$

Note that $R_{e_{j}} I_{\underline{i}}$ is a subspace of the vector space $I_{\underline{i}}+e_{j}$. 
THE DEFINING IDEAL OF POINTS IN MULTI-PROJECTIVE SPACE

For every $\underline{i} \in \mathbb{N}^{k}$, a basis for $R_{\underline{i}}$ as a vector space over $\mathbf{k}$ is the set of all monomials in $R$ of degree $\underline{i}$. Thus, $\operatorname{dim}_{\mathbf{k}} R_{\underline{i}}=\left(\begin{array}{c}n_{1}+i_{1} \\ i_{1}\end{array}\right)\left(\begin{array}{c}n_{2}+i_{2} \\ i_{2}\end{array}\right) \cdots\left(\begin{array}{c}n_{k}+i_{k} \\ i_{k}\end{array}\right)$. We set $N(\underline{i}):=\operatorname{dim}_{\mathbf{k}} R_{\underline{i}}$ for each $\underline{i} \in \mathbb{N}^{k}$.

The $\mathbb{N}^{k}$-graded ring $R$ is the coordinate ring of $\mathbb{P}^{n_{1}} \times \cdots \times \mathbb{P}^{n_{k}}$. If $P \in \mathbb{P}^{n_{1}} \times \cdots \times \mathbb{P}^{n_{k}}$ is a point, and if $I_{P}$ denotes the ideal associated to $P$, then the ideal $I_{P}$ is a prime ideal, and furthermore, $I_{P}=$ $\left(L_{1,1}, \ldots, L_{1, n_{1}}, \ldots, L_{k, 1}, \ldots, L_{k, n_{k}}\right)$ where $\operatorname{deg} L_{i, j}=e_{i}$ for $j=1, \ldots, n_{i}$. Let $P_{1}, \ldots, P_{s}$ be $s$ distinct points in $\mathbb{P}^{n_{1}} \times \cdots \times \mathbb{P}^{n_{k}}$. If $\mathbb{X}=\left\{P_{1}, \ldots, P_{s}\right\}$, then the $\mathbb{N}^{k}$-homogeneous ideal $I_{\mathbb{X}}$ of forms that vanish at $\mathbb{X}$ is $I_{\mathbb{X}}=I_{P_{1}} \cap \cdots \cap I_{P_{s}}$ where $I_{P_{i}}$ is the ideal associated to the point $P_{i}$. The coordinate $\operatorname{ring} R / I_{\mathbb{X}}$ then has the following property.

Lemma 1.1 (16, Lemma 3.3]). If $\mathbb{X} \subseteq \mathbb{P}^{n_{1}} \times \cdots \times \mathbb{P}^{n_{k}}$ is a finite set of points, then for each $l \in[k]$, there exists $L_{l} \in R_{e_{l}}$ such that $\bar{L}_{l}$ is a non-zero divisor in $R / I_{\mathbb{X}}$.

Remark 1.2. After a linear change of variables in the $x_{1, j}$ 's, a change of variables in the $x_{2, j}$ 's, and so on, we can take $L_{l}=x_{l, 0}$ for each $l \in[k]$. We therefore assume, once and for all, that the set of points $\mathbb{X}$ under investigation has the property that $\bar{x}_{l, 0}$ is a non-zero divisor in $R / I_{\mathbb{X}}$ for each $l \in[k]$.

We sometimes write $H_{\mathbb{X}}$ for $H_{R / I_{\mathbb{X}}}$, and call $H_{\mathbb{X}}$ the Hilbert function of $\mathbb{X}$. Classifying the Hilbert functions of sets of points in $\mathbb{P}^{n_{1}} \times \cdots \times \mathbb{P}^{n_{k}}$ with $k \geq 2$ remains an open problem (the case $k=1$ is dealt with in [5]). See [8] and [16] for some progress on this problem. However, some growth conditions on $H_{\mathbb{X}}$ are known.

Theorem 1.3 ([16, Proposition 3.5]). Let $\mathbb{X}$ be a finite set of points in $\mathbb{P}^{n_{1}} \times \cdots \times \mathbb{P}^{n_{k}}$ with Hilbert function $H_{\mathbb{X}}$.

(i) For all $\underline{i} \in \mathbb{N}^{k}, H_{\mathbb{X}}(\underline{i}) \leq H_{\mathbb{X}}\left(\underline{i}+e_{l}\right)$ for all $l \in[k]$.

(ii) If $H_{\mathbb{X}}(\underline{i})=H_{\mathbb{X}}\left(\underline{i}+e_{l}\right)$ for some $l \in[k]$, then $H_{\mathbb{X}}\left(\underline{i}+e_{l}\right)=H_{\mathbb{X}}\left(\underline{i}+2 e_{l}\right)$.

Let $\pi_{i}: \mathbb{P}^{n_{1}} \times \cdots \times \mathbb{P}^{n_{k}} \rightarrow \mathbb{P}^{n_{i}}$ denote the $i$ th projection morphism defined by $P_{1} \times \cdots \times P_{i} \times \cdots \times P_{k} \mapsto P_{i}$. Then $\pi_{i}(\mathbb{X})$ is the set of all the $i$ th-coordinates in $\mathbb{X}$. For each $i \in[k]$, set $t_{i}:=\left|\pi_{i}(\mathbb{X})\right|$. With this notation we have

Theorem 1.4 (16, Corollary 4.7]). Let $\mathbb{X}$ be a finite set of points in $\mathbb{P}^{n_{1}} \times \cdots \times \mathbb{P}^{n_{k}}$ with Hilbert function $H_{\mathbb{X}}$. Fix an $i \in[k]$. If $\left(j_{1}, \ldots, j_{i}, \ldots, j_{k}\right) \in \mathbb{N}^{k}$ with $j_{i} \geq t_{i}-1$, then $H_{\mathbb{X}}\left(j_{1}, \ldots, j_{i}, \ldots, j_{k}\right)=$ $H_{\mathbb{X}}\left(j_{1}, \ldots, t_{i}-1, \ldots, j_{k}\right)$.

Remark 1.5. One can interpret the above results as follows. Fix an integer $i \in[k]$, and fix $k-1$ integers in $\mathbb{N}$, say $j_{1}, \ldots, j_{i-1}, j_{i+1}, \ldots, j_{k}$. Set

$$
\underline{j}_{l}:=\left(j_{1}, \ldots, j_{i-1}, l, j_{i+1}, \ldots, j_{k}\right) \text { for each integer } l \in \mathbb{N} .
$$

Then Theorems 1.3 and 1.4 imply that there exists an integer $l^{\prime} \leq t_{i}-1$ such that the sequence $H_{\mathbb{X}}\left(\underline{j}_{0}\right), H_{\mathbb{X}}\left(\underline{j}_{1}\right), H_{\mathbb{X}}\left(\underline{j}_{2}\right), H_{\mathbb{X}}\left(\underline{j}_{3}\right), \ldots$ has the property that $H_{\mathbb{X}}\left(\underline{j}_{l}\right)<H_{\mathbb{X}}\left(\underline{j}_{l+1}\right)$ if $0 \leq l<l^{\prime}$, but $H_{\mathbb{X}}\left(\underline{j}_{l}\right)=$ $H_{\mathbb{X}}\left(\underline{j}_{l+1}\right)$ if $l \geq l^{\prime}$.

\section{ON THE GENERATORS OF AN IDEAL OF A SET OF POINTS}

Let $I_{\mathbb{X}}$ be the defining ideal of a finite set of points $\mathbb{X} \subseteq \mathbb{P}^{n_{1}} \times \cdots \times \mathbb{P}^{n_{k}}$ with Hilbert function $H_{\mathbb{X}}$. Using only $H_{\mathbb{X}}$, we describe a finite subset $\mathcal{E} \subseteq \mathbb{N}^{k}$ with the property that if $F$ is a generator of $I_{\mathbb{X}}$, then $\operatorname{deg} F \in \mathcal{E}$.

Fix an $l \in[k]$. Then, for each $\underline{j}=\left(j_{1}, \ldots, j_{l-1}, j_{l+1}, \ldots, j_{k}\right) \in \mathbb{N}^{k-1}$, set

$$
i(\underline{j}):=\min \left\{\begin{array}{l|c}
i \in \mathbb{N}^{+} & \begin{array}{c}
H_{\mathbb{X}}\left(j_{1}, \ldots, j_{l-1}, i-1, j_{l+1}, \ldots, j_{k}\right)= \\
H_{\mathbb{X}}\left(j_{1}, \ldots, j_{l-1}, i, j_{l+1}, \ldots, j_{k}\right)
\end{array}
\end{array}\right\} .
$$

The existence of the integer $i(\underline{j})$ follows from Theorem 1.4 
Theorem 2.1. Let $\mathbb{X}$ be a finite set of points of $\mathbb{P}^{n_{1}} \times \cdots \times \mathbb{P}^{n_{k}}$. Fix an $l \in[k]$ and $\underline{j}=\left(j_{1}, \ldots, j_{l-1}, j_{l+1}, \ldots, j_{k}\right) \in$ $\mathbb{N}^{k-1}$. Set $\underline{i}=\left(j_{1}, \ldots, j_{l-1}, i(\underline{j}), j_{l+1}, \ldots, j_{k}\right)$. Then

$$
\left(I_{\mathbb{X}}\right)_{\underline{i}+(r+1) e_{l}}=R_{e_{l}}\left(I_{\mathbb{X}}\right)_{\underline{i}+r e_{l}} \text { for all } r \in \mathbb{N} .
$$

In particular, if there exists $\underline{l} \in \mathbb{N}^{k}$ and $t \in[k]$ such that $H_{\mathbb{X}}(\underline{l})=H_{\mathbb{X}}\left(\underline{l}-e_{t}\right)=H_{\mathbb{X}}\left(\underline{l}-2 e_{t}\right)$, then $I_{\mathbb{X}}$ has no minimal generators of degree $\underline{l}$.

Proof. Without loss of generality, we only consider the case $l=1$. By Remark 1.2 we can take $x_{1,0}$ to be a non-zero divisor. Set $S=\mathbf{k}\left[x_{1,1}, \ldots, x_{k, n_{k}}\right] \cong R /\left(x_{1,0}\right)$ and $\underline{i}=\left(i(\underline{j}), j_{2}, \ldots, j_{k}\right)$ where $\underline{j}=\left(j_{2}, \ldots, j_{k}\right)$.

Because $x_{1,0}$ is a non-zero divisor, $I_{\mathbb{X}} / x_{1,0} I_{\mathbb{X}} \cong\left(\left(I_{\mathbb{X}}, x_{1,0}\right) / x_{1,0}\right)$. For each $\underline{t} \in \mathbb{N}^{k}$ the short exact sequence

$$
0 \longrightarrow\left(I_{\mathbb{X}}\right)_{\underline{t}-e_{1}} \stackrel{\times x_{1} 0}{\longrightarrow}\left(I_{\mathbb{X}}\right)_{\underline{t}} \longrightarrow\left(I_{\mathbb{X}} /\left(x_{1,0} I_{\mathbb{X}}\right)\right)_{\underline{t}} \longrightarrow 0
$$

$\operatorname{implies} \operatorname{dim}_{\mathbf{k}}\left(I_{\mathbb{X}}\right)_{\underline{t}}=\operatorname{dim}_{\mathbf{k}}\left(I_{\mathbb{X}}\right)_{\underline{t}-e_{1}}+\operatorname{dim}_{\mathbf{k}}\left(\left(I_{\mathbb{X}}, x_{1,0}\right) / x_{1,0}\right)_{\underline{t}}$. On the other hand, the short exact sequence

$$
0 \longrightarrow R / I_{\mathbb{X}}\left(-e_{1}\right) \stackrel{\times \bar{x}_{1,0}}{\longrightarrow} R / I_{\mathbb{X}} \longrightarrow R /\left(I_{\mathbb{X}}, x_{1,0}\right) \cong \frac{R /\left(x_{1,0}\right)}{\left(I_{\mathbb{X}}, x_{1,0}\right) / x_{1,0}} \longrightarrow 0,
$$

and the hypothesis that $\operatorname{dim}_{\mathbf{k}}\left(R / I_{\mathbb{X}}\right)_{\underline{i}+r e_{1}}=\operatorname{dim}_{\mathbf{k}}\left(R / I_{\mathbb{X}}\right)_{\underline{i}+(r-1) e_{1}}$ for every $r \in \mathbb{N}$ implies that $\left(\left(I_{\mathbb{X}}, x_{1,0}\right) / x_{1,0}\right)_{\underline{i}+r e_{1}}=$ $\left(R /\left(x_{1,0}\right)\right)_{\underline{i}+r e_{1}} \cong S_{\underline{i}+r e_{1}}$ for all $r \in \mathbb{N}$.

Fix an integer $r \in \mathbb{N}$ and set $W=R_{e_{1}}\left(I_{\mathbb{X}}\right)_{\underline{i}+r e_{1}}$. Then $W \subseteq\left(I_{\mathbb{X}}\right)_{\underline{i}+(r+1) e_{1}}$, and because $x_{1,0}$ is a non-zero divisor

$$
\begin{aligned}
\operatorname{dim}_{\mathbf{k}} W & =\operatorname{dim}_{\mathbf{k}}\left(I_{\mathbb{X}}\right)_{\underline{i}+r e_{1}}+\operatorname{dim}_{\mathbf{k}} W^{\prime} \\
& =\operatorname{dim}_{\mathbf{k}}\left(I_{\mathbb{X}}\right)_{\underline{i}+(r+1) e_{1}}-\operatorname{dim}_{\mathbf{k}} S_{\underline{i}+(r+1) e_{1}}+\operatorname{dim}_{\mathbf{k}} W^{\prime}
\end{aligned}
$$

where $W^{\prime}=\left\{f^{\prime \prime} \mid f=f^{\prime} x_{1,0}+f^{\prime \prime}, f \in W\right\}=\left\{f\left(0, x_{1,1}, \ldots, x_{k, n_{k}}\right) \mid f \in W\right\}$. By slightly abusing notation, $W^{\prime}$ can be viewed as a subset of $S_{\underline{i}+(r+1) e_{1}}$.

It suffices to show that $S_{\underline{i}+(r+1) e_{1}} \subseteq W^{\prime}$ because then $\operatorname{dim}_{\mathbf{k}} S_{\underline{i}+(r+1) e_{1}}=\operatorname{dim}_{\mathbf{k}} W^{\prime}$, whence $\operatorname{dim}_{\mathbf{k}} W=$ $\operatorname{dim}_{\mathbf{k}}\left(I_{\mathbb{X}}\right)_{\underline{i}+(r+1) e_{1}}$ which gives $W=\left(I_{\mathbb{X}}\right)_{\underline{i}+(r+1) e_{1}}$. So, suppose $f \in S_{\underline{i}+(r+1) e_{1}}$. Then $f=f_{1} x_{1,1}+$ $\cdots+f_{n_{1}} x_{1, n_{1}}$ with $f_{i} \in S_{\underline{i}+r e_{1}}$. But $S_{\underline{i}+r e_{1}} \cong\left(\left(I_{\mathbb{X}}, x_{1,0}\right) / x_{1,0}\right)_{\underline{i}+r e_{1}}$, so by abusing notation, there exists $F_{i} \in\left(I_{\mathbb{X}}\right)_{\underline{i}+r e_{1}}$ such that $F_{i}=g_{i} x_{1,0}+f_{i}$. But then $F=F_{1} x_{1,1}+\cdots+F_{n_{1}} x_{1, n_{1}} \in W$, whence $f \in W^{\prime}$.

For the last statement let $\underline{l}=\left(l_{1}, \ldots, l_{k}\right)$ and $i:=i\left(l_{1}, \ldots, l_{t-1}, l_{t+1}, \ldots, l_{k}\right)$. Then $H_{\mathbb{X}}(\underline{l})=H_{\mathbb{X}}\left(\underline{l}-e_{t}\right)=$ $H_{\mathbb{X}}\left(\underline{l}-2 e_{t}\right)$ implies that $\underline{l}-e_{t} \geq\left(l_{1}, \ldots . l_{t-1}, i, l_{t+1}, \ldots, l_{k}\right)$, so $\left(I_{\mathbb{X}}\right)_{\underline{l}}=R_{e_{t}}\left(I_{\mathbb{X}}\right)_{\underline{l}-e_{t}}$.

Let $\mathbb{X}$ be a finite set of points in $\mathbb{P}^{n_{1}} \times \cdots \times \mathbb{P}^{n_{k}}$, and set

$$
\mathcal{B}:=\left\{\underline{i} \in \mathbb{N}^{k} \mid H_{\mathbb{X}}(\underline{i})<\operatorname{dim}_{\mathbf{k}} R_{\underline{i}}=N(\underline{i})\right\}=\left\{\underline{i} \in \mathbb{N}^{k} \mid\left(I_{\mathbb{X}}\right)_{\underline{i}} \neq 0\right\} .
$$

For each $l \in[k]$ and for each $\underline{j}=\left(j_{1}, \ldots, j_{l-1}, j_{l+1}, \ldots, j_{k}\right) \in \mathbb{N}^{k-1}$ set

$$
\mathcal{A}_{l, \underline{j}}:=\left\{\left(j_{1}, \ldots, j_{l-1}, i(\underline{j}), j_{l+1}, \ldots, j_{k}\right)+r e_{l} \mid r \in \mathbb{N}^{+}\right\} .
$$

We then define

$$
\mathcal{A}:=\bigcup_{l=1}^{k}\left(\bigcup_{\underline{j} \in \mathbb{N}^{k-1}} \mathcal{A}_{l, \underline{j}}\right) .
$$

Theorem 2.2. Let $\mathbb{X}$ be a finite set of points in $\mathbb{P}^{n_{1}} \times \cdots \times \mathbb{P}^{n_{k}}$ with defining ideal $I_{\mathbb{X}}$. With the notation as above, set $\mathcal{E}=\mathcal{B} \backslash \mathcal{A}$. Then $\mathcal{E}$ is a finite set. Furthermore, if $f$ is a generator of $I_{\mathbb{X}}$, then $\operatorname{deg} f \in \mathcal{E}$. In particular, $I_{\mathbb{X}}=\left\langle\bigoplus_{\underline{i} \in \mathcal{E}}\left(I_{\mathbb{X}}\right)_{\underline{i}}\right\rangle$. 
Proof. We show that $\mathcal{E}$ is finite. Let $t_{i}:=\left|\pi_{i}(\mathbb{X})\right|$ where $\pi_{i}: \mathbb{P}^{n_{1}} \times \cdots \times \mathbb{P}^{n_{k}} \rightarrow \mathbb{P}^{n_{i}}$ is the $i$ th projection morphism. Set $\mathcal{F}:=\left\{\underline{i} \in \mathbb{N}^{k} \mid \underline{i} \leq\left(t_{1}, t_{2}, \ldots, t_{k}\right)\right\}$. Now suppose that $j=\left(j_{1}, \ldots, j_{k}\right) \in \mathbb{N}^{k} \backslash \mathcal{F}$. Thus, there is a coordinate of $\underline{j}$, say $j_{i}$, such that $j_{i} \geq t_{i}+1$. By Theorem 1.4

$$
H_{\mathbb{X}}(\underline{j})=H_{\mathbb{X}}\left(j_{1}, \ldots, j_{i-1}, t_{i}, j_{i+1}, \ldots, j_{k}\right)=H_{\mathbb{X}}\left(j_{1}, \ldots, j_{i-1}, t_{i}-1, j_{i+1}, \ldots, j_{k}\right) .
$$

This means that $\underline{j} \in \mathcal{A}_{i,\left(j_{1}, \ldots, j_{i-1}, j_{i+1}, \ldots, j_{k}\right)} \subseteq \mathcal{A}$. We thus have $\mathbb{N}^{k} \backslash \mathcal{F} \subseteq \mathcal{A}$. But this implies that $\mathcal{E}=\mathcal{B} \backslash \mathcal{A} \subseteq \mathbb{N}^{k} \backslash \mathcal{A} \subseteq \mathcal{F}$, and since $\mathcal{F}$ is finite, so is $\mathcal{E}$.

For the second statement, let $f$ be a generator of $I_{\mathbb{X}}$. Then it is immediate that $\operatorname{deg} f \in \mathcal{B}$. On the other hand, Theorem 2.1 implies that $\operatorname{deg} f \notin \mathcal{A}_{l, \underline{j}}$ for any $l \in[k]$ or $\underline{j} \in \mathbb{N}^{k-1}$. Hence, $\operatorname{deg} f \notin \mathcal{A}$, so $\operatorname{deg} f \in \mathcal{E}$.

Remark 2.3. We recover Proposition 1.1 (3) of $\left[\right.$ ] when $k=1$, i.e., $\mathbb{X} \subseteq \mathbb{P}^{n}$.

\section{Points in GEneric Position}

Analogous to the definition for points in $\mathbb{P}^{n}$, a set of points in $\mathbb{P}^{n_{1}} \times \cdots \times \mathbb{P}^{n_{k}}$ is said to be in generic position if its Hilbert function is maximal. Although such sets have been studied (cf. [9, 10]) we could find no proof in the literature for the existence of such sets when $k \geq 2$ (the case $k=1$ is [ 6 . Theorem 4]). We therefore begin by providing a proof of this "folklore" result. Then, if $I_{\mathbb{X}}$ is the defining ideal of a set of points in generic position in $\mathbb{P}^{n_{1}} \times \cdots \times \mathbb{P}^{n_{k}}$, we compute the depth of $R / I_{\mathbb{X}}$, and give bounds on the degrees of the generators of $I_{\mathbb{X}}$.

Theorems 1.3 and 1.4 imply the number of possible Hilbert functions for $s$ points is finite. However, since the number of sets with $s$ points is infinite, we can ask if there exists an expected Hilbert function for $s$ points. We give a heuristic argument for this expected function.

If $\left\{m_{1}, \ldots, m_{N(\underline{j})}\right\}$ are the $N(\underline{j})$ monomials of degree $\underline{j}$ in the $\mathbb{N}^{k}$-graded $\operatorname{ring} R$, then any $F \in R_{\underline{j}}$ can be written as $F=\sum_{i=1}^{N(\underline{1})} c_{i} m_{i}$ where $c_{i} \in \mathbf{k}$. Suppose that $P \in \mathbb{P}^{n_{1}} \times \cdots \times \mathbb{P}^{n_{k}}$. For $F \in R_{\underline{j}}$ to vanish at $P$ we require $F(P)=\sum_{i=\overline{1}}^{N(j)} c_{i} m_{i}(P)=0$. By considering the $c_{i}$ 's as unknowns, this equation gives us one linear condition. If $\mathbb{X}=\left\{P_{1}, \ldots, P_{s}\right\}$, then for $F \in R_{\underline{j}}$ to vanish on $\mathbb{X}$ we require that $F\left(P_{1}\right)=\cdots=F\left(P_{s}\right)=0$. We then have a linear system of equations

$$
\left[\begin{array}{ccc}
m_{1}\left(P_{1}\right) & \cdots & m_{N(\underline{j})}\left(P_{1}\right) \\
\vdots & & \vdots \\
m_{1}\left(P_{s}\right) & \cdots & m_{N(\underline{j})}\left(P_{s}\right)
\end{array}\right]\left[\begin{array}{c}
c_{1} \\
\vdots \\
c_{N(\underline{j})}
\end{array}\right]=\left[\begin{array}{c}
0 \\
\vdots \\
0
\end{array}\right] .
$$

The number of linearly independent solutions is the rank of the matrix on the left. For a general enough set of points, we expect this rank to be as large as possible. By [16. Proposition 4.3] the rank of this matrix equals $H_{\mathbb{X}}(\underline{j})$, so we expect a general enough set of $s$ points $\mathbb{X} \subseteq \mathbb{P}^{n_{1}} \times \cdots \times \mathbb{P}^{n_{k}}$ to have the Hilbert function $H_{\mathbb{X}}(\underline{j})=\min \{N(\underline{j}), s\}$ for all $\underline{j} \in \mathbb{N}^{k}$. Proceeding analogously as in the case of points in $\mathbb{P}^{n}$, we make the following definition.

Definition 3.1. Let $\mathbb{X}$ be a finite set of $s$ points in $\mathbb{P}^{n_{1}} \times \cdots \times \mathbb{P}^{n_{k}}$ with Hilbert function $H_{\mathbb{X}}$. If

$$
H_{\mathbb{X}}(\underline{j})=\min \{N(\underline{j}), s\} \quad \text { for all } \underline{j} \in \mathbb{N}^{k},
$$

then the Hilbert function is called maximal. A set of $s$ points is said to be in generic position if its Hilbert function is maximal.

We now show the existence of sets of points in generic position by demonstrating that "most" sets of $s$ points in $\mathbb{P}^{n_{1}} \times \cdots \times \mathbb{P}^{n_{k}}$ are in generic position. We shall denote $\left(\mathbb{P}^{n_{1}} \times \cdots \times \mathbb{P}^{n_{k}}\right) \times \cdots \times\left(\mathbb{P}^{n_{1}} \times \cdots \times \mathbb{P}^{n_{k}}\right)$ $\left(s\right.$ times) by $\left(\mathbb{P}^{n_{1}} \times \cdots \times \mathbb{P}^{n_{k}}\right)^{s}$.

Theorem 3.2. The s-tuples of points of $\mathbb{P}^{n_{1}} \times \cdots \times \mathbb{P}^{n_{k}},\left(P_{1}, \ldots, P_{s}\right)$, considered as points of $\left(\mathbb{P}^{n_{1}} \times\right.$ $\left.\cdots \times \mathbb{P}^{n_{k}}\right)^{s}$, which are in generic position form a non-empty open subset of $\left(\mathbb{P}^{n_{1}} \times \cdots \times \mathbb{P}^{n_{k}}\right)^{s}$. 
Proof. Since the case $k=1$ is found in $\underline{6}$, we can assume that $k \geq 2$. Let $\left\{m_{1}, \ldots, m_{N(\underline{j})}\right\}$ be the $N(\underline{j})$ monomials of degree $\underline{j}=\left(j_{1}, \ldots, j_{k}\right) \in \mathbb{N}^{k}$ in $R$. By composing the product of $j_{i}$-uple embeddings with the Segre embedding we have a morphism $\nu_{\underline{j}}: \mathbb{P}^{n_{1}} \times \cdots \times \mathbb{P}^{n_{k}} \rightarrow \mathbb{P}^{N(\underline{j})-1}$ defined by

$$
P=\left[a_{1,0}: \cdots: a_{1, n_{1}}\right] \times \cdots \times\left[a_{k, 0}: \cdots: a_{k, n_{k}}\right] \longmapsto\left[m_{1}(P): \cdots: m_{N(\underline{j})}(P)\right],
$$

i.e., $m_{i}(P)$ is the monomial $m_{i}$ evaluated at $P$. This induces a morphism

$$
\varphi_{\underline{j}}=\left(\nu_{\underline{j}}: \cdots: \nu_{\underline{j}}\right):\left(\mathbb{P}^{n_{1}} \times \cdots \times \mathbb{P}^{n_{k}}\right)^{s} \longrightarrow\left(\mathbb{P}^{N(\underline{j})-1}\right)^{s}=V_{\underline{j}} .
$$

By [6] there exists a nonempty open subset $W_{\underline{j}} \subseteq V_{\underline{j}}$ with the property that each point $\left(Q_{1}, \ldots, Q_{s}\right) \in W_{\underline{j}}$ corresponds to a set of $s$ points in $\mathbb{P}^{N(\underline{j})-1}$ in generic position. In particular, each point of $W_{\underline{j}}$ corresponds to a set of $s$ points in $\mathbb{P}^{N(\underline{j})-1}$ that impose $\min \{s, N(\underline{j})\}$ conditions on linear forms.

Because $\nu_{j}$ does not vanish everywhere, $U_{j}:=\varphi^{-1}\left(W_{j}\right)$ is a non-empty open subset of $\left(\mathbb{P}^{n_{1}} \times \cdots \times \mathbb{P}^{n_{k}}\right)^{s}$. Furthermore, because $\nu_{j}$ induces an isomorphism between the linear forms of $\mathbb{P}^{N(\underline{j})-1}$ and the forms of $R_{\underline{j}}$, if $\left(P_{1}, \ldots, P_{s}\right) \in U_{\underline{j}}^{-}$, then as a set of points $\mathbb{X}=\left\{P_{1}, \ldots, P_{s}\right\}$ of $\mathbb{P}^{n_{1}} \times \cdots \times \mathbb{P}^{n_{k}}$ we have $H_{\mathbb{X}}(\underline{j})=$ $\min \{s, N(\underline{j})\}$. Hence $\bar{U}=\bigcap_{j \in \mathbb{N}^{k}} U_{\underline{j}}$ consists of those $s$-tuples which correspond to sets of $s$ points in $\mathbb{P}^{n_{1}} \times \cdots \times \mathbb{P}^{n_{k}}$ with maximal Hilbert functions.

To complete the proof, it suffices to show that the intersection $U=\bigcap_{\underline{j} \in \mathbb{N}^{k}} U_{\underline{j}}$ can be taken to be finite, and thus $U$ is open. Suppose $\underline{j} \in \mathbb{N}^{k}$ is such that $s=\min \{s, N(\underline{j})\}$. We claim that $U_{\underline{j}} \subseteq U_{\underline{j}^{\prime}}$ for all $\underline{j} \leq \underline{j}^{\prime}$. Indeed, take $\left(P_{1}, \ldots, P_{s}\right) \in U_{j}$. So, if $\mathbb{X}=\left\{P_{1}, \ldots, P_{s}\right\}$, we have $H_{\mathbb{X}}(\underline{j})=s$. Since the Hilbert function strictly increases until it stabilizes and is bounded by $s, H_{\mathbb{X}}\left(\underline{j}^{\prime}\right)=s<N\left(\underline{j}^{\prime}\right)$ for all $\underline{j} \leq \underline{j^{\prime}}$. Thus $\left(P_{1}, \ldots, P_{s}\right) \in U_{j^{\prime}}$ as desired. Setting $D_{1}=\left\{\underline{j} \in \mathbb{N}^{k} \mid N(\underline{j})<s\right\}$ and $D_{2}=\min \left\{\underline{j} \in \mathbb{N}^{k} \mid N(\underline{j}) \geq s\right\}$, we thus have $D=\bar{D}_{1} \cup D_{2}$ is a finite set and $U=\bigcap_{\underline{j} \in D} U_{\underline{j}}$.

For any finite set of points $\mathbb{X} \subseteq \mathbb{P}^{n_{1}} \times \cdots \times \mathbb{P}^{n_{k}}$, we have $\mathrm{K}$ - $\operatorname{dim} R / I_{\mathbb{X}}=k$. However, it was shown in [17. Proposition 2.6] that the depth of $R / I_{\mathbb{X}}$ may take on any value in $\{1, \ldots, k\}$. When $\mathbb{X}$ is in generic position the depth can be calculated. We begin with a lemma.

Lemma 3.3. Let $n, l \geq 1$ be integers. Then $\left(\begin{array}{c}n+l+1 \\ l+1\end{array}\right) \leq\left(\begin{array}{c}n+l \\ l\end{array}\right)(n+1)$.

Proof. Note that $\left(\begin{array}{c}n+l+1 \\ l+1\end{array}\right)=\left(\begin{array}{c}n+l \\ l\end{array}\right) \cdot \frac{(n+l+1)}{(l+1)}=\left(\begin{array}{c}n+l \\ l\end{array}\right)\left(1+\frac{n}{l+1}\right)$. The inequality now follows since $l \geq 1$, and thus $\left(1+\frac{n}{l+1}\right) \leq(1+n)$.

Theorem 3.4. If $\mathbb{X}$ is a set of $s>1$ points in generic position in $\mathbb{P}^{n_{1}} \times \cdots \times \mathbb{P}^{n_{k}}$, then $\operatorname{depth} R / I_{\mathbb{X}}=1$. In particular, $R / I_{\mathbb{X}}$ is Cohen-Macaulay if and only if $k=1$.

Proof. By Lemma 1.1 depth $R / I_{\mathbb{X}} \geq 1$. We show that equality holds. Without loss of generality, take $n_{1} \leq n_{2} \leq \cdots \leq n_{k}$ and let $l$ be the minimal integer such that $\left(\begin{array}{c}n_{1}+l \\ l\end{array}\right) \geq s$. Since $\mathbb{X}$ is in generic position, $H_{\mathbb{X}}(l, 0, \ldots, 0)=\min \left\{\left(\begin{array}{c}n_{1}+l \\ l\end{array}\right), s\right\}=s$.

Claim. If $\underline{j} \in \mathbb{N}^{k}$ and $\underline{j}>(l-1,0, \ldots, 0)$, then $H_{\mathbb{X}}(\underline{j})=s$.

Proof of the Claim. If $j_{1}>l-1$, then $\left(\begin{array}{c}n_{1}+j_{1} \\ j_{1}\end{array}\right) \geq\left(\begin{array}{c}n_{1}+l \\ l\end{array}\right)$. Thus $N(\underline{j}) \geq\left(\begin{array}{c}n_{1}+l \\ l\end{array}\right) \geq \min \left\{\left(\begin{array}{c}n_{1}+l \\ l\end{array}\right), s\right\}=s$, and hence, $H_{\mathbb{X}}(j)=s$. So, suppose $j_{1}=l-1$. Since $j>(l-1,0, \ldots, 0)$, there exists $m \in\{2, \ldots, k\}$ such that $j_{m}>0$. Since $n_{1} \leq n_{m}$, we have the following inequalities: $N(\underline{j}) \geq\left(\begin{array}{c}n_{1}+l-1 \\ l-1\end{array}\right)\left(\begin{array}{c}n_{m}+j_{m} \\ j_{m}\end{array}\right) \geq\left(\begin{array}{c}n_{1}+l-1 \\ l-1\end{array}\right)\left(\begin{array}{c}n_{1}+1 \\ 1\end{array}\right)$. By Lemma 3.3 we also have $\left(\begin{array}{c}n_{1}+l-1 \\ l-1\end{array}\right)\left(n_{1}+1\right) \geq\left(\begin{array}{c}n_{1}+l \\ l\end{array}\right)$. Hence, $N(\underline{j}) \geq\left(\begin{array}{c}n_{1}+l \\ l\end{array}\right) \geq \min \left\{\left(\begin{array}{c}n_{1}+l \\ l\end{array}\right), s\right\}=s$. Therefore, $H_{\mathbb{X}}(\underline{j})=s$, as desired. 
Since $\bar{x}_{1,0}$ is a non-zero divisor of $R / I_{\mathbb{X}}$ we have the short exact sequence

$$
0 \longrightarrow\left(R / I_{\mathbb{X}}\right)\left(-e_{1}\right) \stackrel{\times \bar{x}_{1}, 0}{\longrightarrow} R / I_{\mathbb{X}} \longrightarrow R /\left(I_{\mathbb{X}}, x_{1,0}\right)=R / J \longrightarrow 0
$$

where $J=\left(I_{\mathbb{X}}, x_{1,0}\right)$. Thus the Hilbert function of $R / J$ is $H_{R / J}(\underline{j})=H_{\mathbb{X}}(\underline{j})-H_{\mathbb{X}}\left(\underline{j}-e_{1}\right)$ for all $\underline{j} \in \mathbb{N}^{k}$, where $H_{\mathbb{X}}(\underline{j})=0$ if $\underline{j} \geq \underline{0}$. From the claim, it follows that if $\underline{j}>(\bar{l}, 0, \ldots, 0)$, then

$$
H_{R / J}(\underline{j})=H_{\mathbb{X}}(\underline{j})-H_{\mathbb{X}}\left(\underline{j}-e_{1}\right)=s-s=0 .
$$

On the other hand, if $\underline{j}=(l, 0, \ldots, 0)$, then

$$
H_{R / J}(\underline{j})=H_{\mathbb{X}}\left(l e_{1}\right)-H_{\mathbb{X}}\left((l-1) e_{1}\right)=s-\left(\begin{array}{c}
n_{1}+l-1 \\
l-1
\end{array}\right)>0 .
$$

Since $s \neq 1$ there exists a non-constant element $F \in R_{l e_{1}}$ such that $0 \neq \bar{F} \in R / J$.

It suffices to demonstrate that all the non-constant homogeneous elements of $R / J$ are annihilated by $\bar{F}$, and hence, $\operatorname{depth} R / J=0$. So, suppose that $G \in R$ is such that $0 \neq \bar{G} \in R / J$. Without loss of generality we can take $G$ to be an $\mathbb{N}^{k}$-homogeneous element with $\operatorname{deg} G=\left(j_{1}, \ldots, j_{k}\right)>\underline{0}$. Now $\operatorname{deg} F G=\left(j_{1}+l, j_{2}, \ldots, j_{k}\right)>(l, 0, \ldots, 0)$. Since $H_{R / J}\left(j_{1}+l, j_{2}, \ldots, j_{k}\right)=0$, it follows that $F G \in J$.

Remark 3.5. If $s=1$, then $\operatorname{depth} R / I_{\mathbb{X}}=k$ because the ideal of a point is a complete intersection.

We now apply Theorem[2.2 to describe the degrees of the generators of $I_{\mathbb{X}}$ when $\mathbb{X}$ is in generic position. We introduce some notation: if $E=\left\{\underline{j}_{1}, \ldots, \underline{j}_{l}\right\} \subseteq \mathbb{N}^{k}$, and $\underline{i} \in \mathbb{N}^{k}$, then

$$
E+\underline{i}:=\left\{\underline{j}_{1}+\underline{i}, \underline{j}_{2}+\underline{i}, \ldots, \underline{j}_{l}+\underline{i}\right\} \subseteq \mathbb{N}^{k} .
$$

Also, let $\mathcal{D}:=\min \left\{\underline{i} \in \mathbb{N}^{k} \mid\left(I_{\mathbb{X}}\right)_{\underline{i}} \neq 0\right\}=\min \left\{\underline{i} \in \mathbb{N}^{k} \mid H_{\mathbb{X}}(\underline{i})<N(\underline{i})\right\}$. (Recall that min $\mathcal{S}$ with $\mathcal{S} \subseteq \mathbb{N}^{k}$ is the set of minimal elements of $\mathcal{S}$ with respect to the partial ordering $\underline{i} \geq \underline{j}$ if $i_{h} \geq j_{j}$ for all $h$.) When $\mathbb{X}$ is a set of $s$ points in generic position we have $\mathcal{D}=\min \left\{\underline{i} \in \mathbb{N}^{k} \mid s<N(\underline{i})\right\}$.

Theorem 3.6. Let $I_{\mathbb{X}}$ be the defining ideal of a set of $s$ points in $\mathbb{P}^{n_{1}} \times \cdots \times \mathbb{P}^{n_{k}}$ in generic position, and set $\mathcal{T}=\mathcal{D} \cup\left(\bigcup_{i=1}^{k} \mathcal{D}+e_{i}\right)$. If $f$ is a generator of $I_{\mathbb{X}}$, then $\operatorname{deg} f \in \mathcal{T}$. In particular, $I_{\mathbb{X}}=\left\langle\bigoplus_{\underline{i} \in \mathcal{T}}\left(I_{\mathbb{X}}\right)_{\underline{i}}\right\rangle$.

Proof. The proof consists of two parts.

Step 1. We use Theorem 2.2 to show that $I_{\mathbb{X}}=\left\langle\bigoplus_{\underline{i} \in \mathcal{T}^{\prime}}\left(I_{\mathbb{X}}\right)_{\underline{i}}\right\rangle$ where

$$
\mathcal{T}^{\prime}=\bigcup_{\left\{l_{1}, \ldots, l_{t}\right\} \in \mathcal{P}([k])} \mathcal{D}+\left(e_{l_{1}}+\cdots+e_{l_{t}}\right)
$$

and $\mathcal{P}([k])$ denotes the power set of $[k]$. It is enough to show that $\mathcal{E} \subseteq \mathcal{T}^{\prime}$. To do this, we need to first show that $\mathcal{D} \subseteq \mathcal{E}$. If $\underline{i} \in \mathcal{D}$, then $H_{\mathbb{X}}\left(\underline{i}-2 e_{l}\right)<H_{\mathbb{X}}\left(\underline{i}-e_{l}\right) \leq H_{\mathbb{X}}(\underline{i})=s$ for all $l \in[k]$. So $\underline{i} \in \mathcal{D} \subseteq \mathcal{B}$, but $\underline{i} \notin \mathcal{A}$, hence $\underline{i} \in \mathcal{E}$.

Suppose $\underline{j} \in \mathcal{E}$. Then there exists $\underline{i} \in \mathcal{D} \subseteq \mathcal{E}$ such that $\underline{j} \geq \underline{i}$. We can thus write $\underline{j}=\left(i_{1}+m_{1}, \ldots, i_{k}+\right.$ $m_{k}$ ) where $\underline{\underline{i}}=\left(i_{1}, \ldots, i_{k}\right)$. If $m_{1}=\cdots=m_{k}=0$, then $\underline{j}=\underline{i}$ and hence $\underline{j} \in \mathcal{E}$. So, suppose $m_{l} \geq 1$ for some $l \in[k]$. If $m_{l} \geq 2$, then $H_{\mathbb{X}}(\underline{j})=H_{\mathbb{X}}\left(\underline{j}-e_{l}\right)=\bar{H}_{\mathbb{X}}\left(\underline{j}-2 e_{l}\right)=s$ since $\underline{j}-2 e_{l} \geq \underline{i}$. But then $\underline{j} \in \mathcal{A}$, so $\underline{j} \notin \mathcal{E}$. Hence, for each $l \in[k], m_{l}=0$ or 1 . So, if $m_{l_{1}}=\cdots=m_{l_{t}}=1$, and 0 otherwise, then $\bar{j}=\underline{i}+\left(e_{l_{1}}+\cdots+e_{l_{t}}\right) \in \mathcal{T}^{\prime}$.

Step 2. If $F$ is a generator of $I_{\mathbb{X}}$ with $\operatorname{deg} F=\underline{j}$, then the previous step implies there exists $\underline{i} \in \mathcal{D}$ such that $\underline{j}=\underline{i}+e_{l_{1}}+\cdots+e_{l_{t}}$ for some subset $\left\{l_{1}, \ldots, l_{t}\right\} \subseteq[k]$. We wish to show that $t=0$ or 1 , i.e., $\operatorname{deg} \bar{F}=\underline{i}$, or $\operatorname{deg} F=\underline{i}+e_{l}$ from some $l \in[k]$.

Let $\underline{i} \in \mathcal{D}$ and let $\left\{l_{1}, \ldots, l_{t}\right\}$ be any subset of $[k]$ with $t \geq 2$. Set $\underline{j}=\underline{i}+e_{l_{1}}+\cdots+e_{l_{t}}$. If we can show that $\left(I_{\mathbb{X}}\right)_{j}=R_{e_{l_{1}}}\left(I_{\mathbb{X}}\right)_{j-e_{l_{1}}}+R_{e_{l_{2}}}\left(I_{\mathbb{X}}\right)_{\underline{j}-e_{l_{2}}}$ then we shall be finished because this implies that $\left(I_{\mathbb{X}}\right)_{j}$ contains no new generators. 
By Remark $1.2 \bar{x}_{l_{2}, 0}$ is a non-zero divisor. Set $S=\mathbf{k}\left[x_{1,0}, \ldots, \widehat{x}_{l_{2}, 0}, \ldots, x_{k, n_{k}}\right] \cong R /\left(x_{l_{2}, 0}\right)$. For each $\underline{t} \in \mathbb{N}^{k}$ we have the short exact sequence of vector spaces:

$$
0 \longrightarrow\left(I_{\mathbb{X}}\right)_{\underline{t}-e_{l_{2}}} \stackrel{\times x_{l_{2}, 0}}{\longrightarrow}\left(I_{\mathbb{X}}\right)_{\underline{t}} \longrightarrow\left(I_{\mathbb{X}} / x_{l_{2}, 0} I_{\mathbb{X}}\right)_{\underline{t}} \cong\left(\left(I_{\mathbb{X}}, x_{l_{2}, 0}\right) /\left(x_{l_{2}, 0}\right)\right)_{\underline{t}} \longrightarrow 0 .
$$

This gives $\operatorname{dim}_{\mathbf{k}}\left(I_{\mathbb{X}}\right)_{\underline{t}}=\operatorname{dim}_{\mathbf{k}}\left(I_{\mathbb{X}}\right)_{\underline{t}-e_{l_{2}}}+\operatorname{dim}_{\mathbf{k}}\left(\left(I_{\mathbb{X}}, x_{l_{2}, 0}\right) /\left(x_{l_{2}, 0}\right)\right)_{\underline{t}}$.

Since $\mathbb{X}$ is in generic position, $H_{\mathbb{X}}(\underline{j})=H_{\mathbb{X}}\left(\underline{j}-e_{l_{2}}\right)=H_{\mathbb{X}}\left(\underline{j}-e_{l_{1}}\right)=H_{\mathbb{X}}\left(\underline{j}-e_{l_{1}}-e_{l_{2}}\right)=s$. Thus, we can use the short exact sequence

$$
0 \longrightarrow R / I_{\mathbb{X}}\left(-e_{l_{2}}\right) \stackrel{\times \bar{x}_{l_{2}, 0}}{\longrightarrow} R / I_{\mathbb{X}} \longrightarrow R /\left(I_{\mathbb{X}}, x_{l_{2}, 0}\right) \cong \frac{R /\left(x_{l_{2}, 0}\right)}{\left(I_{\mathbb{X}}, x_{l_{2}, 0}\right) /\left(x_{l_{2}, 0}\right)} \longrightarrow 0
$$

to show that $\left(\left(I_{\mathbb{X}}, x_{l_{2}, 0}\right) /\left(x_{l_{2}, 0}\right)\right)_{\underline{j}-e_{l_{1}}} \cong S_{\underline{j}-e_{l_{1}}}$ and $\left(\left(I_{\mathbb{X}}, x_{l_{2}, 0}\right) /\left(x_{l_{2}, 0}\right)\right)_{\underline{j}} \cong S_{\underline{j}}$.

Set $W=R_{e_{l_{1}}}\left(I_{\mathbb{X}}\right)_{j-e_{l_{1}}}+R_{e_{l_{2}}}\left(I_{\mathbb{X}}\right)_{j-e_{l_{2}}}$. So $W \subseteq\left(I_{\mathbb{X}}\right)_{j}$. Because $x_{l_{2}, 0}$ is a non-zero divisor

$$
\operatorname{dim}_{\mathbf{k}} W=\operatorname{dim}_{\mathbf{k}}\left(I_{\mathbb{X}}\right)_{\underline{j}-e_{l_{2}}}+\operatorname{dim}_{\mathbf{k}} W^{\prime}=\operatorname{dim}_{\mathbf{k}}\left(I_{\mathbb{X}}\right)_{\underline{j}}-\operatorname{dim}_{\mathbf{k}} S_{\underline{j}}+\operatorname{dim}_{\mathbf{k}} W^{\prime}
$$

where $W^{\prime}=\left\{f^{\prime \prime} \mid f=f^{\prime} x_{l_{2}, 0}+f^{\prime \prime}, f \in W\right\}$. The vector space $W^{\prime}$ can be viewed as a subspace of $S_{j}$. It now suffices to show that $S_{\underline{j}} \subseteq W^{\prime}$ because then $\operatorname{dim}_{\mathbf{k}} W=\operatorname{dim}_{\mathbf{k}}\left(I_{\mathbb{X}}\right)_{\underline{j}}$, and thus, $W=\left(I_{\mathbb{X}}\right)_{\underline{j}}$. So, let $f \in S_{\underline{j}}$. Then $f=f_{0} x_{l_{1}, 0}+\cdots+f_{n_{l_{1}}} x_{l_{1}, n_{l_{1}}}$ with $f_{i} \in S_{\underline{j}-e_{l_{1}}}$. Since $S_{\underline{j}-e_{l_{1}}}^{-} \cong\left(\left(I_{\mathbb{X}}, x_{l_{2}, 0}\right) /\left(x_{l_{2}, 0}\right)\right)_{\underline{j}-e_{l_{1}}}$, there exists (with a slight abuse of notation) $F_{i} \in\left(I_{\mathbb{X}}\right)_{\underline{j}-e_{l_{1}}}$ such that $F_{i}=g_{i} x_{l_{2}, 0}+f_{i}$. But then $F_{0} x_{l_{1}, 0}+\cdots+F_{n_{l_{1}}} x_{l_{1}, n_{l_{1}}} \in W$, and hence, $f \in W^{\prime}$.

Remark 3.7. If $k=1$ and $\mathbb{X}$ is a set of $s$ points in generic position, then we obtain the well known result that $I_{\mathbb{X}}=\left\langle I_{d} \oplus I_{d+1}\right\rangle$ where $d=\min \left\{i \mid\left(\begin{array}{c}n+i \\ i\end{array}\right)>s\right\}$. 9] Lemma 4.2] is a proof of this theorem in the special case that $\mathbb{X}$ is a set of points in generic position in $\mathbb{P}^{1} \times \mathbb{P}^{1}$. If $\mathbb{X}$ is a set of points in generic position in $\mathbb{P}^{n_{1}} \times \cdots \times \mathbb{P}^{n_{k}}$, and if we set $\left.d_{i}:=\min \left\{d \mid \begin{array}{c}n_{i}+d \\ d\end{array}\right) \geq|\mathbb{X}|\right\}$ and $D:=\max \left\{d_{1}+1, \ldots, d_{k}+1\right\}$, then the above result implies that $I_{\mathbb{X}}$, considered as an $\mathbb{N}^{1}$-graded ideal of $R$, is generated by forms of degree $\leq D$. This is extended in 13 to show that $\operatorname{reg}\left(I_{\mathbb{X}}\right)=D$, where $\operatorname{reg}\left(I_{\mathbb{X}}\right)$ is the Castelnuovo-Mumford regularity of $I_{\mathbb{X}}$.

Corollary 3.8. Let $\mathbb{X}$ be a set of $s$ points in generic position in $\mathbb{P}^{n_{1}} \times \cdots \times \mathbb{P}^{n_{k}}$ with Hilbert function $H_{\mathbb{X}}$. If there exists $l, m \in[k]$ (not necessarily distinct) and $\underline{j} \in \mathbb{N}^{k}$ such that $H_{\mathbb{X}}(\underline{j})=H_{\mathbb{X}}\left(\underline{j}-e_{l}\right)=$ $H_{\mathbb{X}}\left(\underline{j}-e_{l}-e_{m}\right)=s$, then $\left(I_{\mathbb{X}}\right)_{\underline{j}}$ contains no generators of $I_{\mathbb{X}}$.

Proof. If $l=m$, then this is simply Theorem 2.1 If $l \neq m$, then $H_{\mathbb{X}}\left(\underline{j}-e_{m}\right)=s$ because $\underline{j}-e_{l}-e_{m} \leq \underline{j}-e_{m}$ and $\mathbb{X}$ is in generic position. Arguing as in Step 2 of Theorem 3.6 we have $\left(I_{\mathbb{X}}\right)_{\underline{j}}=R_{e_{l}}\left(I_{\mathbb{X}}\right)_{\underline{j}-e_{l}}+$ $R_{e_{m}}\left(I_{\mathbb{X}}\right)_{\underline{j}-e_{m}}$.

\section{ON THE VALUE OF $\nu\left(I_{\mathbb{X}}\right)$ FOR POINTS IN GENERIC POSITION}

In this section we study $\nu\left(I_{\mathbb{X}}\right)$, the minimal number of generators of $I_{\mathbb{X}}$, when $\mathbb{X}$ is a set of points in generic position. Unless specified otherwise, the set of points under consideration will be non-degenerate, that is, $|\mathbb{X}|>\max \left\{n_{1}, \ldots, n_{k}\right\}$. We give an upper bound on $\nu\left(I_{\mathbb{X}}\right)$ that can be calculated from $n_{1}, \ldots, n_{k}$ and $|\mathbb{X}|=s$. We also show that calculating $\nu\left(I_{\mathbb{X}}\right)$ is equivalent to calculating the dimensions of specific vector spaces. In some special cases, we are able to compute these dimensions.

So, suppose $\mathbb{X}$ is a non-degenerate set of $s$ points in generic position in $\mathbb{P}^{n_{1}} \times \cdots \times \mathbb{P}^{n_{k}}$. By Theorem [3.6] if $\underline{i} \in \mathcal{D}=\min \left\{\underline{i} \in \mathbb{N}^{k} \mid s<N(\underline{i})\right\}=\min \left\{\underline{i} \mid\left(I_{\mathbb{X}}\right)_{\underline{i}} \neq 0\right\}$, by degree considerations $\left(I_{\mathbb{X}}\right)_{\underline{i}}$ cannot be generated by elements of smaller degree. So the $\operatorname{dim}_{\mathbf{k}}\left(I_{\mathbb{X}}\right)_{\underline{i}}=N(\underline{i})-H_{\mathbb{X}}(\underline{i})$ linearly independent elements of $\left(I_{\mathbb{X}}\right)_{\underline{i}}$ must be generators of $I_{\mathbb{X}}$. This gives a crude bound on $\nu\left(I_{\mathbb{X}}\right)$ :

$$
\nu\left(I_{\mathbb{X}}\right) \geq \sum_{\underline{i} \in \mathcal{D}} \operatorname{dim}_{\mathbf{k}}\left(I_{\mathbb{X}}\right)_{\underline{i}}=\sum_{\underline{i} \in \mathcal{D}}(N(\underline{i})-s) .
$$


By Theorem 3.6 to compute $\nu\left(I_{\mathbb{X}}\right)$ it suffices to calculate the number of generators of $I_{\mathbb{X}}$ in $\left(I_{\mathbb{X}}\right)_{\underline{j}}$ for each $\underline{j} \in \bigcup_{l=1}^{k}\left(\mathcal{D}+e_{l}\right)$.

We wish to describe a subset of $\bigcup_{l=1}^{k}\left(\mathcal{D}+e_{l}\right)$ such that for each $\underline{j}$ in this subset, $\left(I_{\mathbb{X}}\right)_{j}$ contains no new generators of $I_{\mathbb{X}}$. We introduce some suitable notation. For each $\underline{i} \in \mathcal{D}$ set

$$
\mathbb{D}_{\underline{i}}:=\left\{\underline{j} \in \mathbb{N}^{k} \mid \underline{j} \geq \underline{i}\right\} \backslash\left\{\underline{i}, \underline{i}+e_{1}, \underline{i}+e_{2}, \ldots, \underline{i}+e_{k}\right\} .
$$

If follows that $\underline{j} \in \mathbb{D}_{\underline{i}}$ if and only if $\underline{j}-e_{l_{1}}-e_{l_{2}} \geq \underline{i}$ for some not necessarily distinct $l_{1}, l_{2} \in[k]$.

Lemma 4.1. Let $\mathbb{X}$ be a non-degenerate set of s points in $\mathbb{P}^{n_{1}} \times \cdots \times \mathbb{P}^{n_{k}}$. With the notation as above, suppose $\underline{j} \in\left[\bigcup_{l=1}^{k}\left(\mathcal{D}+e_{l}\right)\right] \cap\left[\bigcup_{\underline{i} \in \mathcal{D}} \mathbb{D}_{\underline{i}}\right]$. Then $I_{\mathbb{X}}$ has no generator of degree $\underline{j}$.

Proof. Since $\underline{j} \in \mathbb{D}_{\underline{i}}$ for some $\underline{i} \in \mathcal{D}, \underline{j}-e_{l_{1}}-e_{l_{2}} \geq \underline{i}$ for some not necessarily distinct $l_{1}, l_{2} \in[k]$. Because $\mathbb{X}$ is in generic position, we have $H_{\mathbb{X}}\left(\underline{j}-e_{l_{1}}-e_{l_{2}}\right)=H_{\mathbb{X}}\left(\underline{j}-e_{l_{1}}\right)=H_{\mathbb{X}}(\underline{j})=s$, and so the conclusion follows from Corollary 3.8

Set

$$
\mathbb{D}:=\left[\bigcup_{l=1}^{k}\left(\mathcal{D}+e_{l}\right)\right] \backslash\left[\bigcup_{\underline{i} \in \mathcal{D}} \mathbb{D}_{\underline{i}}\right] .
$$

Because of Lemma 4.1 to determine $\nu\left(I_{\mathbb{X}}\right)$ it is enough to count the number of generators of $I_{\mathbb{X}}$ with degree $\underline{j} \in \mathbb{D}$.

So, let $\underline{j} \in \mathbb{D}$. Since $\underline{j} \in \bigcup_{l=1}^{k}\left(\mathcal{D}+e_{l}\right)$, we can associate to $\underline{j}$ a unique subset $L_{j}:=\left\{l_{1}, \ldots, l_{t}\right\} \subseteq[k]$ such that $\underline{j} \in \overline{\mathcal{D}}+e_{l_{m}}$ for each $l_{m} \in L_{\underline{j}}$ but $\underline{j} \notin \mathcal{D}+e_{l}$ if $l \in[k] \backslash L_{\underline{j}}$. For each $l_{m} \in L_{\underline{j}}$ there then exists a unique $\underline{i}_{l_{m}} \in \mathcal{D}$ such that $\underline{j}=\underline{i}_{l_{m}}+e_{l_{m}}$. So, for each $l_{m} \in L_{\underline{j}}$ we can define $W_{l_{m}, \underline{i}_{l_{m}}}:=R_{e_{l_{m}}}\left(I_{\mathbb{X}}\right)_{\underline{i}_{l_{m}}} \subseteq\left(I_{\mathbb{X}}\right)_{\underline{j}}$. For each $\underline{j} \in \mathbb{D}$ we set

$$
W_{\underline{j}}:=W_{l_{1}, \underline{i}_{l_{1}}}+\cdots+W_{l_{t}, \underline{i}_{l_{t}}}=\sum_{l_{m} \in L_{\underline{j}}} W_{l_{m}, \underline{i}_{l_{m}}} \subseteq\left(I_{\mathbb{X}}\right)_{\underline{j}} .
$$

Thus $W_{\underline{j}}$ is the subvector space of $\left(I_{\mathbb{X}}\right)_{\underline{j}}$ that consists of all the forms in $I_{\mathbb{X}}$ of degree $\underline{j}$ that come from forms of lower degree in $I_{\mathbb{X}}$. The number of new generators of $I_{\mathbb{X}}$ of degree $\underline{j}$ with $\underline{j} \in \overline{\mathbb{D}}$ is then

$$
\operatorname{dim}_{\mathbf{k}}\left(I_{\mathbb{X}}\right)_{\underline{j}}-\operatorname{dim}_{\mathbf{k}}\left(W_{l_{1}, \underline{i}_{l_{1}}}+\cdots+W_{l_{t}, \underline{i}_{l_{t}}}\right)=N(\underline{j})-s-\operatorname{dim}_{\mathbf{k}} W_{\underline{j}} .
$$

We summarize this discussion with the following theorem.

Theorem 4.2. Let $\mathbb{X}$ be a non-degenerate set of $s$ points in generic position in $\mathbb{P}^{n_{1}} \times \cdots \times \mathbb{P}^{n_{k}}$. With the notation as above

$$
\begin{aligned}
\nu\left(I_{\mathbb{X}}\right) & =\sum_{\underline{i} \in \mathcal{D}} \operatorname{dim}_{\mathbf{k}}\left(I_{\mathbb{X}}\right)_{\underline{i}}+\sum_{\underline{j} \in \mathbb{D}}\left(\operatorname{dim}_{\mathbf{k}}\left(I_{\mathbb{X}}\right)_{\underline{j}}-\operatorname{dim}_{\mathbf{k}} W_{\underline{j}}\right) \\
& =\sum_{\underline{i} \in \mathcal{D}}(N(\underline{i})-s)+\sum_{\underline{j} \in \mathbb{D}}\left(N(\underline{j})-s-\operatorname{dim}_{\mathbf{k}} W_{\underline{j}}\right) .
\end{aligned}
$$

Computing $\nu\left(I_{\mathbb{X}}\right)$ is thus equivalent to computing $\operatorname{dim}_{\mathbf{k}} W_{\underline{j}}$ for each $\underline{j} \in \mathbb{D}$. Arguing as in [7] Proposition 7] one has the following lower bounds:

Lemma 4.3. Suppose $\underline{j} \in \mathbb{D}$ and $L_{\underline{j}}=\left\{l_{1}, \ldots, l_{t}\right\}$. For every $l_{m} \in L_{\underline{j}}$

$$
\operatorname{dim}_{\mathbf{k}} W_{\underline{j}} \geq \operatorname{dim}_{\mathbf{k}} W_{l_{m}}, \underline{i}_{l_{m}} \geq 2 \operatorname{dim}_{\mathbf{k}}\left(I_{\mathbb{X}}\right)_{\underline{i}_{l_{m}}} .
$$

Combining Lemma 4.3 with Theorem 4.2 gives us an upper bound on $\nu\left(I_{\mathbb{X}}\right)$. 
Corollary 4.4. Let $\mathbb{X}$ be a non-degenerate set of $s$ points in generic position in $\mathbb{P}^{n_{1}} \times \cdots \times \mathbb{P}^{n_{k}}$. With the notation as above

$$
\nu\left(I_{\mathbb{X}}\right) \leq \sum_{\underline{i} \in \mathcal{D}}(N(\underline{i})-s)+\sum_{\underline{j} \in \mathbb{D}}\left(N(\underline{j})-2 N\left(\underline{j}-e_{l_{1}}\right)+s\right)
$$

where $l_{1} \in L_{\underline{j}}=\left\{l_{1}, \ldots, l_{t}\right\}$ for $\underline{j} \in \mathbb{D}$.

Proof. For each $\underline{j} \in \mathbb{D}$, let $l_{1} \in L_{\underline{j}}$. Then $\operatorname{dim}_{\mathbf{k}} W_{\underline{j}} \geq 2 \operatorname{dim}_{\mathbf{k}}\left(I_{\mathbb{X}}\right)_{\underline{j}-e_{l_{1}}}=2 N\left(\underline{j}-e_{l_{1}}\right)-2 s$. Now use Theorem 4.2 .

In general, calculating $\operatorname{dim}_{\mathbf{k}} W_{\underline{j}}$ for each $\underline{j} \in \mathbb{D}$ is a very difficult problem. Indeed, if $L_{\underline{j}}=\left\{l_{1}, \ldots, l_{t}\right\}$, then there is no a priori formula for calculating $\operatorname{dim}_{\mathbf{k}} W_{l_{m}, \underline{i}_{l_{m}}}=\operatorname{dim}_{\mathbf{k}}\left(R_{e_{l_{m}}}\left(I_{\mathbb{X}}\right)_{\underline{i}_{l_{m}}}\right)$ for each $l_{m} \in L_{\underline{j}}$. The problem is further complicated when $\left|L_{\underline{j}}\right|=t \geq 2$ because then we need to know how $W_{l_{m}, \underline{i}_{l_{m}}}$ and $W_{l_{n}, \underline{i}_{l_{n}}}$ intersect in $\left(I_{\mathbb{X}}\right)_{\underline{j}}$ for each $l_{n}, l_{m} \in L_{\underline{j}}$.

However, under some extra hypotheses on either $s=|\mathbb{X}|$ or $n_{1}, \ldots, n_{k}$ we can be quite explicit about $\operatorname{dim}_{\mathbf{k}} W_{\underline{j}}$ for some $\underline{j} \in \mathbb{D}$. The remaining results of this section are of this vein.

Lemma 4.5. Let $\mathbb{X} \subseteq \mathbb{P}^{1} \times \cdots \times \mathbb{P}^{1}$ be any finite set of points. Then, for any $\underline{i} \in \mathbb{N}^{k}$ and $l \in[k]$,

$$
\operatorname{dim}_{\mathbf{k}}\left(R_{e_{l}}\left(I_{\mathbb{X}}\right)_{\underline{i}}\right)=2 \operatorname{dim}_{\mathbf{k}}\left(I_{\mathbb{X}}\right)_{\underline{i}}-\operatorname{dim}_{\mathbf{k}}\left(I_{\mathbb{X}}\right)_{\underline{i}-e_{l}}
$$

where $\operatorname{dim}_{\mathbf{k}}\left(I_{\mathbb{X}}\right)_{\underline{i}-e_{l}}=0$ if $\underline{i}-e_{l} \nsupseteq 0$.

Proof. The proof for the case $\mathbb{X} \subseteq \mathbb{P}^{1} \times \mathbb{P}^{1}$ given in [8] Lemma 2.3] can be extended to this case.

Theorem 4.6. Let $\mathbb{X} \subseteq \mathbb{P}^{1} \times \cdots \times \mathbb{P}^{1}$ be a set of $s>1$ points in generic position. With the notation as above, suppose $\underline{j} \in \mathbb{D}$ with $L_{\underline{j}}=\{l\}$. Then

$$
\operatorname{dim}_{\mathbf{k}} W_{\underline{j}}= \begin{cases}\operatorname{dim}_{\mathbf{k}}\left(I_{\mathbb{X}}\right)_{j}=N(\underline{j})-s & \text { if } N\left(\underline{j}-2 e_{l}\right)=s . \\ 2 \operatorname{dim}_{\mathbf{k}}\left(I_{\mathbb{X}}\right)_{\underline{j}-e_{l}}=2 N\left(\underline{j}-e_{l}\right)-2 s & \text { if } N\left(\underline{j}-2 e_{l}\right)<s .\end{cases}
$$

Proof. The hypothesis $L_{j}=\{l\}$ implies $\underline{j}-e_{l}=\underline{i} \in \mathcal{D}$ but $\underline{j}-e_{m} \notin \mathcal{D}$ for any $m \in[k] \backslash\{l\}$. Hence $\operatorname{dim}_{\mathbf{k}} W_{\underline{j}}=\operatorname{dim}_{\mathbf{k}}\left(R_{e_{l}}\left(I_{\mathbb{X}}\right)_{\underline{i}}\right)$. Since $\underline{i} \in \mathcal{D}$, there does not exists an $\underline{i}^{\prime} \in \mathcal{D}$ such that $\underline{i}-e_{l} \geq \underline{i}^{\prime}$, and hence $\operatorname{dim}_{\mathbf{k}}\left(I_{\mathbb{X}}\right)_{\underline{i}-e_{l}}=0$. By Lemma 4.5 we thus have $\operatorname{dim}_{\mathbf{k}} W_{j}=2 \operatorname{dim}_{\mathbf{k}}\left(I_{\mathbb{X}}\right)_{\underline{i}}=2 N\left(\underline{j}-e_{l}\right)-2 s$. The reader can now verify that $2 N\left(\underline{j}-e_{l}\right)-2 s=N(\underline{j})-s$ if $s=N\left(\underline{j}-2 e_{l}\right)$.

Theorem 4.7. Let $\mathbb{X}$ be a non-degenerate set of s points in generic position in $\mathbb{P}^{n_{1}} \times \cdots \times \mathbb{P}^{n_{k}}$ and $\underline{j} \in \mathbb{D}$.

(i) If there exists $l \in[k]$ such that $N\left(\underline{j}-2 e_{l}\right)=s$, then $\operatorname{dim}_{\mathbf{k}} W_{\underline{j}}=\operatorname{dim}_{\mathbf{k}}\left(I_{\mathbb{X}}\right)_{\underline{j}}$.

(ii) If $L_{\underline{j}}=\{l\}$ and $s=N\left(\underline{j}-e_{l}\right)-1$, then $\operatorname{dim}_{\mathbf{k}} W_{\underline{j}}=n_{l}+1$.

Proof. (i) Since $N\left(\underline{j}-2 e_{l}\right)=s, H_{\mathbb{X}}\left(\underline{j}-2 e_{l}\right)=H_{\mathbb{X}}\left(\underline{j}-e_{l}\right)=s$. Now apply Theorem 2.1

(ii) We are given that $\underline{j}-e_{l} \in \mathcal{D}$ and $\operatorname{dim}_{\mathbf{k}}\left(I_{\mathbb{X}}\right)_{j-e_{l}}=N\left(\underline{j}-e_{l}\right)-s=1$. So let $F$ be a basis for $\left(I_{\mathbb{X}}\right)_{\underline{j}-e_{l}}$. Then $x_{l, 0} F, \ldots, \bar{x}_{l, n_{l}} F$ form a linearly independent basis of $W_{\underline{j}}=R_{e_{l}}\left(I_{\mathbb{X}}\right)_{\underline{j}-e_{l}}$.

\section{ON THE EXPeCted VAlue OF $\nu\left(I_{\mathbb{X}}\right)$}

Let $\mathbb{X}$ be a non-degenerate set of points in generic position in $\mathbb{P}^{n_{1}} \times \cdots \times \mathbb{P}^{n_{k}}$. In this section we are interested in determining if there is an expected value for $\nu\left(I_{\mathbb{X}}\right)$. After showing that $\nu\left(I_{\mathbb{X}}\right)$ is constant on some open subset of $\left(\mathbb{P}^{n_{1}} \times \cdots \times \mathbb{P}^{n_{k}}\right)^{s}$, we give a lower bound for this value. When $k=1$ the resulting lower bound is conjectured to equal $\nu\left(I_{\mathbb{X}}\right)$ on some non-empty open subset of $\left(\mathbb{P}^{n}\right)^{s}$ by the Ideal Generation Conjecture. Therefore, it seems natural to expect that our generalized lower bound equals $\nu\left(I_{\mathbb{X}}\right)$ on some non-empty open subset of $\left(\mathbb{P}^{n_{1}} \times \cdots \times \mathbb{P}^{n_{k}}\right)^{s}$, thus generalizing the IGC to points in $\mathbb{P}^{n_{1}} \times \cdots \times \mathbb{P}^{n_{k}}$. However, although we have found computationally that in many cases $\nu\left(I_{\mathbb{X}}\right)$ equals the 
lower bound, we show that there exist $s$ and $n_{1}, \ldots, n_{k}$ for which $\nu\left(I_{\mathbb{X}}\right)$ is always larger than this bound. We continue to use the notation of the previous sections.

If $\left(P_{1}, \ldots, P_{s}\right) \in\left(\mathbb{P}^{n_{1}} \times \cdots \times \mathbb{P}^{n_{k}}\right)^{s}$ is such that $P_{1}, \ldots, P_{s}$ are distinct points, then we shall write $I\left(P_{1}, \ldots, P_{s}\right)$ to denote the defining ideal of $\left\{P_{1}, \ldots, P_{s}\right\} \subseteq \mathbb{P}^{n_{1}} \times \cdots \times \mathbb{P}^{n_{k}}$. Furthermore, if $j \in \mathcal{D}$ with $L_{\underline{j}}=\left\{l_{1}, \ldots, l_{t}\right\}$, then we write $W\left(P_{1}, \ldots, P_{s}\right)_{\underline{j}}$ for the vector space $W\left(P_{1}, \ldots, P_{s}\right)_{\underline{j}}:=R_{e_{l_{1}}} I_{\underline{j}}-e_{l_{1}}+\cdots+$ $\overline{R_{e_{l_{t}}}} I_{\underline{j}}-e_{l_{t}} \subseteq I_{\underline{j}}$ where $I=I\left(P_{1}, \ldots, P_{s}\right)$.

Theorem 5.1. Let $s>\max \left\{n_{1}, \ldots, n_{k}\right\}$. Then there exists an open set $U \subseteq\left(\mathbb{P}^{n_{1}} \times \cdots \times \mathbb{P}^{n_{k}}\right)^{s}$ such that if $\left(P_{1}, \ldots, P_{s}\right) \in U$, then $\operatorname{dim}_{\mathbf{k}} W\left(P_{1}, \ldots, P_{s}\right)_{\underline{j}}$ is the maximum possible for all $\underline{j} \in \mathbb{D}$. In particular, $\nu\left(I\left(P_{1}, \ldots, P_{s}\right)\right)$ is constant for all $\left(P_{1}, \ldots, P_{s}\right) \in \bar{U}$.

Proof. It is enough to show that for each $\underline{j} \in \mathbb{D}$, there exists an open subset $U_{j} \subseteq\left(\mathbb{P}^{n_{1}} \times \cdots \times \mathbb{P}^{n_{k}}\right)^{s}$ with the property that if $\left(P_{1}, \ldots, P_{s}\right) \in U_{\underline{j}}$, then $\operatorname{dim}_{\mathbf{k}} W\left(P_{1}, \ldots, P_{s}\right)_{\underline{j}}$ is maximal. Then, since $|\mathbb{D}|<\infty$, the desired open set is $U=\bigcap_{\underline{j} \in \mathbb{D}} U_{\underline{j}}$.

So, let $\underline{j} \in \mathbb{D}$ and suppose $L_{\underline{j}}=\left\{l_{1}, \ldots, l_{t}\right\}$. For each $l_{m} \in L_{\underline{j}}$ set $\underline{i}_{l_{m}}:=\underline{j}-e_{l_{m}}$. Let $W \subseteq$ $\left(\mathbb{P}^{n_{1}} \times \cdots \times \mathbb{P}^{n_{k}}\right)^{s}$ denote the open set of Theorem 3.2 consisting of the $s$ distinct points in generic position. Then, using the proof of the claim found after Remark 2.8 in 4, we can show that there exists an open set $U_{l_{m}} \subseteq W$ such that for all $\left(P_{1}, \ldots, P_{s}\right) \in U_{l_{m}}, \operatorname{dim}_{\mathbf{k}} R_{e_{l_{m}}} I\left(P_{1}, \ldots, P_{s}\right)_{\underline{i}_{l_{m}}}$ is the maximum possible.

If we let $G_{l_{m}, 1}, \ldots, G_{l_{m}, N\left(\underline{i}_{l_{m}}\right)-s}$ denote the $N\left(\underline{i}_{l_{m}}\right)-s=\operatorname{dim}_{\mathbf{k}} I\left(P_{1}, \ldots, P_{s}\right)_{\underline{i}_{l_{m}}}$ distinct basis elements of $I\left(P_{1}, \ldots, P_{s}\right)_{\underline{i}_{m}}$, then the elements

$$
\left\{x_{l_{m}, i} G_{l_{m}, j} \mid 0 \leq i \leq n_{l_{m}}, 1 \leq j \leq N\left(\underline{i}_{l_{m}}\right)-s\right\}
$$

generate $R_{e_{l_{m}}} I\left(P_{1}, \ldots, P_{s}\right)_{\underline{i}_{l_{m}}}$. Set $M_{l_{m}}=\left(n_{l_{m}}+1\right)\left(N\left(\underline{i}_{l_{m}}\right)-s\right)$ and form the $M_{l_{m}} \times N(\underline{j})$ matrix $\mathcal{M}_{l_{m}}$ which expresses how the $x_{l_{m}, i} G_{l_{m}, j}$ 's are linear combinations of the $N(\underline{j})$ monomials of degree $\underline{j}$. Since $\operatorname{rank} \mathcal{M}_{l_{m}}=\operatorname{dim}_{\mathbf{k}} R_{e_{l_{m}}} I\left(P_{1}, \ldots, P_{s}\right)_{\underline{i}_{l_{m}}}$, this rank is maximal for all $\left(P_{1}, \ldots, P_{s}\right) \in U_{l_{m}}$.

Let $\mathcal{M}$ be the $\left(\sum_{l_{m} \in L_{\underline{j}}} M_{l_{m}}\right) \times N(\underline{j})$ matrix $\mathcal{M}:=\left[\begin{array}{c}\mathcal{M}_{l_{1}} \\ \vdots \\ \mathcal{M}_{l_{t}}\end{array}\right]$. Then the rank of $\mathcal{M}$ is equal to $\operatorname{dim}_{\mathbf{k}} W\left(P_{1}, \ldots, P_{s}\right)_{j}$. The rank of $\mathcal{M}$ will therefore assume its maximal value on some open subset $U_{\underline{j}} \subseteq \bigcap_{l_{m} \in L_{\underline{j}}} U_{l_{m}}$. This is the desired set $U_{\underline{j}}$.

We can give a lower bound on $\nu\left(I_{\mathbb{X}}\right)$ by using Theorem 4.2 and bounds on $\operatorname{dim}_{\mathbf{k}} W_{\underline{j}}$ for each $\underline{j} \in \mathbb{D}$. For each $l_{m} \in L_{\underline{j}}$ the dimension of $W_{l_{m}, \underline{i}_{l_{m}}}$ is bounded by

$$
\operatorname{dim}_{\mathbf{k}} W_{l_{m}, \underline{i}_{l_{m}}} \leq \min \left\{\operatorname{dim}_{\mathbf{k}}\left(I_{\mathbb{X}}\right)_{\underline{j}},\left(n_{l_{m}}+1\right) \operatorname{dim}_{\mathbf{k}}\left(I_{\mathbb{X}}\right)_{\underline{i}_{l_{m}}}\right\} .
$$

Furthermore, if $l_{m}, l_{n} \in L_{\underline{j}}$ and $l_{m} \neq l_{n}$, then

$$
\operatorname{dim}_{\mathbf{k}}\left(W_{l_{m}, \underline{l}_{l_{m}}}+W_{l_{n}, \underline{l}_{l_{n}}}\right) \leq \operatorname{dim}_{\mathbf{k}} W_{l_{m}, \underline{i}_{l_{m}}}+\operatorname{dim}_{\mathbf{k}} W_{l_{n}, \underline{i}_{l_{n}}}
$$

We thus arrive at the following upper bound for $W_{\underline{j}}$ :

$$
\operatorname{dim}_{\mathbf{k}} W_{\underline{j}} \leq \min \left\{\operatorname{dim}_{\mathbf{k}}\left(I_{\mathbb{X}}\right)_{\underline{j}}, \sum_{l_{m} \in L_{\underline{j}}}\left(n_{l_{m}}+1\right) \operatorname{dim}_{\mathbf{k}}\left(I_{\mathbb{X}}\right)_{\underline{i}_{l_{m}}}\right\} .
$$

Since the values of $\operatorname{dim}_{\mathbf{k}}\left(I_{\mathbb{X}}\right)_{\underline{j}}$ and $\operatorname{dim}_{\mathbf{k}}\left(I_{\mathbb{X}}\right)_{\underline{i}_{m}}$ are known because $\mathbb{X}$ is in generic position, combing the above upper bound with Proposition 4.2 results in the following lower bound: 
Theorem 5.2. Let $\mathbb{X}$ be a non-degenerate set of $s$ points in generic position in $\mathbb{P}^{n_{1}} \times \cdots \times \mathbb{P}^{n_{k}}$, and set

$$
v\left(s ; n_{1}, \ldots, n_{k}\right):=\sum_{\underline{i} \in \mathcal{D}}(N(\underline{i})-s)+\sum_{\underline{j} \in \mathbb{D}} \max \left\{0, N(\underline{j})-s-\sum_{l_{m} \in L_{\underline{j}}}\left(n_{l_{m}}+1\right)\left(N\left(\underline{i}_{l_{m}}\right)-s\right)\right\} .
$$

Then $\nu\left(I_{\mathbb{X}}\right) \geq v=v\left(s ; n_{1}, \ldots, n_{k}\right)$.

Remark 5.3. If $k=1$, then

$$
v=v(s ; n)=\left(\begin{array}{c}
d+n \\
n
\end{array}\right)-s+\max \left\{0,\left(\begin{array}{c}
d+1+n \\
n
\end{array}\right)-s-(n+1)\left(\left(\begin{array}{c}
d+n \\
n
\end{array}\right)-s\right)\right\}
$$

where $d=\min \left\{i \mid\left(\begin{array}{c}n+i \\ i\end{array}\right)>s\right\}$. The Ideal Generation Conjecture conjectures that $\nu\left(I_{\mathbb{X}}\right)=v$ on some non-empty open subset of $\left(\mathbb{P}^{n}\right)^{s}$. Although known to be true in some cases (for $n=2$ see [4], for $n=3$ see [1, and for $s \gg n$ see 14]) the conjecture remains open in general. The conjecture was formulated using the heuristic argument that "generically" $\operatorname{dim}_{\mathbf{k}} W_{\underline{j}}$ should be as large as possible, thus implying equality in the bounds (1) and (2). It seems natural to extend this heuristic argument to points in $\mathbb{P}^{n_{1}} \times \cdots \times \mathbb{P}^{n_{k}}$ to generalize the Ideal Generation Conjecture by expecting that $\nu\left(I_{\mathbb{X}}\right)=v\left(s ; n_{1}, \ldots, n_{k}\right)$ for some non-empty open set of $\left(\mathbb{P}^{n_{1}} \times \cdots \times \mathbb{P}^{n_{k}}\right)^{s}$. But as we show at the end of this section, sometimes $\nu\left(I_{\mathbb{X}}\right)>v$ if $k \geq 2$.

In 9 10 Giuffrida, et al. computed the minimal free resolution of points in generic position in $\mathbb{P}^{1} \times \mathbb{P}^{1}$, and in particular, showed that $\nu\left(I_{\mathbb{X}}\right)=v$ on some non-empty open set of $\left(\mathbb{P}^{1} \times \mathbb{P}^{1}\right)^{s}$. Since their notation and approach is different than ours, for the convenience of the reader we make this connection more transparent.

Theorem 5.4. There exists a non-empty open subset $U \subseteq\left(\mathbb{P}^{1} \times \mathbb{P}^{1}\right)^{s}$ with $s \geq 2$ such that for all $\left(P_{1}, \ldots, P_{s}\right) \in U$, the points $\left\{P_{1}, \ldots, P_{s}\right\}$ are in generic position, and $\nu\left(I\left(P_{1}, \ldots, P_{s}\right)\right)=v(s ; 1,1)$.

Proof. It suffices to show that the bound (2) for $\operatorname{dim}_{\mathbf{k}} W_{\underline{j}}$ is in fact an equality for each $\underline{j} \in \mathbb{D}$. If $\underline{j}=(i, j) \in \mathbb{D}$, there are two possibilities: $\left|L_{j}\right|=1$ or 2 . In the former, by Theorem 4.6 we have equality in (2).

For the second case, by [10, Theorem 4.3] there exists a non-empty subset $U \subseteq\left(\mathbb{P}^{1} \times \mathbb{P}^{1}\right)^{s}$ such that for all $\underline{j}=(i, j) \in \mathbb{D}$ with $\left|L_{\underline{j}}\right|=2$ and for each $\left(P_{1}, \ldots, P_{s}\right) \in U$, we have

$$
\operatorname{dim}_{\mathbf{k}}\left(I\left(P_{1}, \ldots, P_{s}\right)\right)_{i, j}-\operatorname{dim}_{\mathbf{k}} W\left(P_{1}, \ldots, P_{s}\right)_{i, j}=\max \left\{0,-d_{i, j}\right\} .
$$

Here, $d_{i, j}$ is the $(i, j)$ th entry of what [10] call the second difference Hilbert matrix of $\mathbb{X}=\left\{P_{1}, \ldots, P_{s}\right\}$ which is computed from the Hilbert function on $\mathbb{X}$. Since $\left|L_{\underline{j}}\right|=2$ and because $\mathbb{X}$ is in generic position, $H_{\mathbb{X}}$, written as a matrix, has the form

\begin{tabular}{r|cccc} 
& & $(j-2)$ & $(j-1)$ & $j$ \\
\hline & & $\vdots$ & $\vdots$ & $\vdots$ \\
$(i-2)$ & $\cdots$ & $(i-1)(j-1)$ & $(i-1) j$ & $(i-1)(j+1)$ \\
$(i-1)$ & $\cdots$ & $i(j-1)$ & $i j$ & $s$ \\
$i$ & $\cdots$ & $(i+1)(j-1)$ & $s$ & $s$
\end{tabular}

This local description of the Hilbert function, and the definition of $d_{i, j}$ on page 422 of 10 gives

$$
\begin{aligned}
-d_{i, j} & =[(i+1)(j+1)-s]-[2((i+1) j-s)+2((j+1) i-s)] \\
& =\operatorname{dim}_{\mathbf{k}}\left(I_{\mathbb{X}}\right)_{i, j}-2 \operatorname{dim}_{\mathbf{k}}\left(I_{\mathbb{X}}\right)_{i-1, j}-2 \operatorname{dim}_{\mathbf{k}}\left(I_{\mathbb{X}}\right)_{i, j-1} .
\end{aligned}
$$

We thus have equality in (2).

We now show that $\nu\left(I_{\mathbb{X}}\right)$ may not equal $v=v\left(s ; n_{1}, \ldots, n_{k}\right)$ in general. We begin by showing that any example of points $\mathbb{X}$ with $\nu\left(I_{\mathbb{X}}\right)>v$ can be extended to an infinite family of examples. 
Lemma 5.5. Suppose that for every non-degenerate set $\mathbb{X}$ of $s$ points in generic position in $\mathbb{P}^{n_{1}} \times \cdots \times \mathbb{P}^{n_{k}}$ we have $\nu\left(I_{\mathbb{X}}\right)>v\left(s ; n_{1}, \ldots, n_{k}\right)$. If $\mathbb{X}^{\prime}$ is any non-degenerate set of $s$ points in generic position in $\mathbb{P}^{n_{1}} \times \cdots \times \mathbb{P}^{n_{k}} \times \mathbb{P}^{m_{1}} \times \cdots \times \mathbb{P}^{m_{l}}$, then $\nu\left(I_{\mathbb{X}^{\prime}}\right)>v\left(s ; n_{1}, \ldots, n_{k}, m_{1}, \ldots, m_{l}\right)$.

Proof. Let $\mathbb{X}^{\prime}$ be a set of $s$ points in generic position in $\mathbb{P}^{n_{1}} \times \cdots \times \mathbb{P}^{n_{k}} \times \mathbb{P}^{m_{1}} \times \cdots \times \mathbb{P}^{m_{l}}$. Let $I_{\mathbb{X}^{\prime}}$ be the associated ideal and set $I:=\bigoplus_{\left(i_{1}, \ldots, i_{k}\right) \in \mathbb{N}^{k}}\left(I_{\mathbb{X}^{\prime}}\right)_{\left(i_{1}, \ldots, i_{k}, 0, \ldots, 0\right)}$. Then $I$ is isomorphic to an ideal $I_{\mathbb{X}} \subseteq \mathbf{k}\left[x_{1,0}, \ldots, x_{1, n_{1}}, \ldots, x_{k, 0}, \ldots, x_{k, n_{k}}\right]$ where $I_{\mathbb{X}}$ is the defining ideal of a set $\mathbb{X}$ of $s$ points in generic position in $\mathbb{P}^{n_{1}} \times \cdots \times \mathbb{P}^{n_{k}}$.

By hypothesis, there exists $\underline{j}=\left(j_{1}, \ldots, j_{k}\right) \in \mathbb{N}^{k}$ such that $\left(I_{\mathbb{X}}\right)_{j}$ contains a generator that has not been accounted for by $v\left(s ; n_{1}, \ldots, n_{k}\right)$. Hence $\left(I_{\mathbb{X}^{\prime}}\right)_{\left(j_{1}, \ldots, j_{k}, 0, \ldots, 0\right)} \cong\left(\bar{I}_{\mathbb{X}}\right)_{j}$ contains a generator of $I_{\mathbb{X}^{\prime}}$ that is not expected, and thus $\nu\left(I_{\mathbb{X}^{\prime}}\right)$ will be strictly larger than $v\left(s ; n_{1}, \ldots, n_{k}, m_{1}, \ldots, m_{l}\right)$.

We now give a case where $\nu\left(I_{\mathbb{X}}\right)$ fails to agree with the lower bound.

Theorem 5.6. Let $\mathbb{X}$ be three points in generic position in $\mathbb{P}^{1} \times \mathbb{P}^{1} \times \mathbb{P}^{1}$. Then $\nu\left(I_{\mathbb{X}}\right)>v(3 ; 1,1,1)$.

Proof. It is enough to show the existence of some $\underline{j} \in \mathbb{D}$ for which we have a strict inequality in (2) for $\operatorname{dim}_{\mathbf{k}} W_{j}$. Now $\{(1,1,0),(1,0,1),(0,1,1)\} \subseteq \mathcal{D}=\overline{\min }\left\{\underline{i} \in \mathbb{N}^{3} \mid N(\underline{i})>3\right\}$, and so $\underline{j}:=(1,1,1) \in \mathbb{D}$ with $L_{\underline{j}}=\{1,2,3\}$. The expected dimension of $W_{\underline{j}}$ is

$$
\min \left\{\operatorname{dim}_{\mathbf{k}}\left(I_{\mathbb{X}}\right)_{\underline{j}}, \sum_{i \in\{1,2,3\}} 2 \operatorname{dim}_{\mathbf{k}}\left(I_{\mathbb{X}}\right)_{\underline{j}}-e_{i}\right\}=\min \left\{8-3, \sum_{i \in\{1,2,3\}} 2(4-3)\right\}=5,
$$

or equivalently, we expect $\left(I_{\mathbb{X}}\right)_{\underline{j}}$ to contain no generators.

However, we claim $\operatorname{dim}_{\mathbf{k}} W_{\underline{j}} \leq 4$, and hence, $\left(I_{\mathbb{X}}\right)_{\underline{j}}$ contains a new generator. Let $P_{1}, P_{2}, P_{3}$ be the distinct points of $\mathbb{X}$, and after a linear change of variables in each set of coordinates, we can assume $P_{1}=[1: 0] \times[1: 0] \times[1: 0], P_{2}=\left[1: a_{1}\right] \times\left[1: a_{2}\right] \times\left[1: a_{3}\right]$, and $P_{3}=\left[1: b_{1}\right] \times\left[1: b_{2}\right] \times\left[1: b_{3}\right]$ with $a_{i} \neq b_{i}$ for $i=1,2,3$. Because $\mathbb{X}$ is in generic position $\operatorname{dim}_{\mathbf{k}}\left(I_{\mathbb{X}}\right)_{1,1,0}=\operatorname{dim}_{\mathbf{k}}\left(I_{\mathbb{X}}\right)_{1,0,1}=\operatorname{dim}_{\mathbf{k}}\left(I_{\mathbb{X}}\right)_{0,1,1}=1$. To find a basis for each of these vector spaces, it suffices to find a form of the proper degree in $I_{\mathbb{X}}$. From our description of the points we can find such forms:

$$
\begin{aligned}
& F_{1}:=\left(a_{2} b_{1}-a_{1} b_{2}\right) x_{1} y_{1}+a_{2} b_{2}\left(a_{1}-b_{1}\right) x_{1} y_{0}+a_{1} b_{1}\left(b_{2}-a_{2}\right) x_{0} y_{1} \in\left(I_{\mathbb{X}}\right)_{1,1,0} \\
& F_{2}:=\left(a_{3} b_{1}-a_{1} b_{3}\right) x_{1} z_{1}+a_{3} b_{3}\left(a_{1}-b_{1}\right) x_{1} z_{0}+a_{1} b_{1}\left(b_{3}-a_{3}\right) x_{0} z_{1} \in\left(I_{\mathbb{X}}\right)_{1,0,1} \\
& F_{3}:=\left(a_{2} b_{3}-a_{3} b_{2}\right) y_{1} z_{1}+a_{3} b_{3}\left(b_{2}-a_{2}\right) y_{1} z_{0}+a_{2} b_{2}\left(a_{3}-b_{3}\right) y_{0} z_{1} \in\left(I_{\mathbb{X}}\right)_{0,1,1} .
\end{aligned}
$$

It follows that $z_{0} F_{1}, z_{1} F_{1}, y_{0} F_{2}, y_{1} F_{2}, x_{0} F_{3}, x_{1} F_{3}$ generate the vector space $W_{\underline{j}}$. A routine calculation will now verify that

$$
\begin{aligned}
a_{1} b_{1}\left(a_{1}-b_{1}\right) x_{0} F_{3}= & \left(a_{1}-b_{1}\right)\left[a_{3} b_{3} z_{0} F_{1}-a_{2} b_{2} y_{0} F_{2}\right]+\left(a_{3} b_{1}-a_{1} b_{3}\right) z_{1} F_{1} \\
& -\left(a_{2} b_{1}-b_{2} a_{1}\right) y_{1} F_{2} \\
\left(a_{1}-b_{1}\right) x_{1} F_{3}= & \left(b_{2}-a_{2}\right) y_{1} F_{2}+\left(a_{3}-b_{3}\right) z_{1} F_{1} .
\end{aligned}
$$

Thus, $x_{0} F_{3}, x_{1} F_{3}$ are in the vector space spanned by $z_{0} F_{1}, z_{1} F_{1}, y_{0} F_{2}, y_{1} F_{2}$, whence $\operatorname{dim}_{\mathbf{k}} W_{\underline{j}} \leq 4<5=$ the expected dimension.

With this result we can construct examples with $\nu\left(I_{\mathbb{X}}\right)$ arbitrarily larger than $v\left(s ; n_{1}, \ldots, n_{k}\right)$.

Corollary 5.7. Let $\mathbb{X}$ be three points in generic position in $\mathbb{P}^{1} \times \cdots \times \mathbb{P}^{1}(k \geq 3$ times $)$. Then $\nu\left(I_{\mathbb{X}}\right) \geq$ $v(3 ; 1, \ldots, 1)+\left(\begin{array}{c}k \\ 3\end{array}\right)$.

Proof. There are $\left(\begin{array}{l}k \\ 3\end{array}\right)$ tuples $\underline{i} \in \mathbb{N}^{k}$ which have exactly three 1 's and $k-3$ zeroes. Let $\underline{i}$ be such a tuple, and suppose that the three 1 's are in $i_{1}$ th, $i_{2}$ th, and $i_{3}$ th position. If $\pi_{i_{1}, i_{2}, i_{3}}: \mathbb{P}^{1} \times \cdots \times \mathbb{P}^{1} \rightarrow \mathbb{P}^{1} \times \mathbb{P}^{1} \times \mathbb{P}^{1}$ is the projection map onto the $i_{1}$ th, $i_{2}$ th, and $i_{3}$ th coordinates, then $\mathbb{Y}=\pi_{i_{1}, i_{2}, i_{3}}(\mathbb{X}) \subseteq \mathbb{P}^{1} \times \mathbb{P}^{1} \times \mathbb{P}^{1}$ is a set of three points in generic position. Hence, $\left(I_{\mathbb{X}}\right)_{\underline{i}} \cong\left(I_{\mathbb{Y}}\right)_{1,1,1}$. But by Theorem $\left[5.6\left(I_{\mathbb{Y}}\right)_{1,1,1}\right.$ contains 
at least one generator not accounted for by $v(3 ; 1,1,1)$, and thus, $\left(I_{\mathbb{X}}\right)_{\underline{i}}$ has a generator not counted by $v(3 ; 1, \ldots, 1)$.

Using CoCoA [3] we have computed $\nu\left(I_{\mathbb{X}}\right)$ in the following ranges:

$$
\begin{array}{lll}
k=2 & 1 \leq n_{1} \leq n_{2} \leq 5 & n_{2}<s \leq 20 \\
k=3 & 1 \leq n_{1} \leq n_{2} \leq n_{3} \leq 5 & n_{3}<s \leq 10 \\
k=4 & 1 \leq n_{1} \leq n_{2} \leq n_{3} \leq n_{4} \leq 5 & n_{4}<s \leq 10
\end{array}
$$

Besides the example of Theorem 5.6 (and those examples that are a consequence of Lemma [5.5) we found that

$$
\nu\left(I_{\mathbb{X}}\right)>v(1+n+n ; 1+n+n) \text { for } 1 \leq n \leq 7 .
$$

From this data it appears that $\nu\left(I_{\mathbb{X}}\right)>v(1+n+n ; 1, n, n)$ for all $n$. Notice that the example of Theorem [5.6 is also part of this family. Using CoCoA we found that in each of these cases $\operatorname{dim}_{\mathbf{k}} W_{1,1,1}$ is smaller than the expected dimension.

We point out, however, that in every other case the computed value of $\nu\left(I_{\mathbb{X}}\right)$ agrees with $v\left(s ; n_{1}, \ldots, n_{k}\right)$. These computations leads us to believe that $\nu\left(I_{\mathbb{X}}\right)=v\left(s ; n_{1}, \ldots, n_{k}\right)$ for a large number $s$ and $n_{1}, \ldots, n_{k}$. Moreover, we know of no counterexamples when $k \leq 2$. We conclude by giving some questions inspired by our computer examples.

Question 5.8. For $s=(1+n+n)$ points in generic position in $\mathbb{P}^{1} \times \mathbb{P}^{n} \times \mathbb{P}^{n}$ is $\nu\left(I_{\mathbb{X}}\right)$ always the larger $v(s ; 1, n, n)$ ? Is this family of examples the only family where the lower bound fails to hold? If not, can we classify all $s$ and $n_{1}, \ldots, n_{k}$ for which $\nu\left(I_{\mathbb{X}}\right) \neq v\left(s ; n_{1}, \ldots, n_{k}\right)$ ? Does the lower bound value always hold in the case $k \leq 2$ ? How should a generalized Ideal Generalization Conjecture be formulated to account for these examples?

\section{REFERENCES}

[1] E. Ballico, Generators for homogeneous ideals of $s$ general points in $\mathbb{P}^{3}$. J. Algebra 106 (1987) 46-52.

[2] M.V. Catalisano, A.V. Geramita, A. Gimigliano, Rank of tensors, secant varieties of Segre varieties and fat points. Linear Alg. and its Appl. 355 (2002) 263-284.

[3] CoCoATeam, CoCoA: a system for doing Computations in Commutative Algebra, Available at http://cocoa.dima.unige.it

[4] A.V. Geramita, P. Maroscia, The ideal of forms vanishing at a finite set of points in $\mathbb{P}^{n}$. J. Algebra 90 (1984) 528-555.

[5] A.V. Geramita, P. Maroscia, L. Roberts, The Hilbert function of a reduced $k$-algebra. J. London Math. Soc. 28 (1983) 443-452.

[6] A.V. Geramita, F. Orecchia, On the Cohen-Macaulay type of $s$-lines in $A^{n+1}$. J. Algebra 70 (1981) $116-140$.

[7] A.V. Geramita, F. Orecchia, Minimally generating ideals defining certain tangent cones. J. Algebra 78 (1982) 36-57.

[8] S. Giuffrida, R. Maggioni, A. Ragusa, On the postulation of 0-dimensional subschemes on a smooth quadric. Pacific J. Math. 155 (1992) 251-282.

[9] S. Giuffrida, R. Maggioni, A. Ragusa, Resolutions of 0-dimensional subschemes of a smooth quadric. In Zerodimensional schemes (Ravello, 1992), de Gruyter, Berlin (1994) 191-204.

[10] S. Giuffrida, R. Maggioni, A. Ragusa, Resolutions of generic points lying on a smooth quadric. Manuscripta Math. 91 (1996) 421-444.

[11] E. Guardo, Fat point schemes on a smooth quadric. J. Pure Appl. Algebra 162 (2001) $183-208$.

[12] E. Guardo, A. Van Tuyl, Fat Points in $\mathbb{P}^{1} \times \mathbb{P}^{1}$ and their Hilbert functions. Canad. J. Math. 56 (2004) $716-741$.

[13] H. Tài Hà, A. Van Tuyl, The regularity of points in multi-projective spaces. J. Pure Appl. Algebra 187 (2004) $153-167$.

[14] A. Hirschowitz, C. Simpson, La résolution minimale de l'idéal d'un arrangement général d'un grand nombre de points dans $\mathbb{P}^{n}$. Invent. Math. 126 (1996) 467-503.

[15] P. Roberts, Multiplicities and Chern Classes in Local Algebra, Cambridge Tracts in Mathematics, 133. Cambridge University Press, Cambridge, 1998.

[16] A. Van Tuyl, The border of the Hilbert function of a set of points in $\mathbb{P}^{n_{1}} \times \cdots \times \mathbb{P}^{n_{k}}$. J. Pure Appl. Algebra $\mathbf{1 7 6}(2002)$ $223-247$.

[17] A. Van Tuyl, The Hilbert functions of ACM sets of points in $\mathbb{P}^{n_{1}} \times \cdots \times \mathbb{P}^{n_{k}}$. J. Algebra $264(2003) 420-441$.

Department of Mathematical Sciences, Lakehead University, Thunder Bay, ON P7B 5E1, Canada

E-mail address: avantuyl@sleet.lakeheadu.ca 